\title{
Semantic interference through multiple distractors in picture naming \\ in people with aphasia
}

\author{
Cornelia van Scherpenberg ${ }^{1,2,3}{ }^{*}$, Rasha Abdel Rahman ${ }^{1,4}$, \\ Frank Regenbrecht ${ }^{3}$, Hellmuth Obrig 1,2,3
}

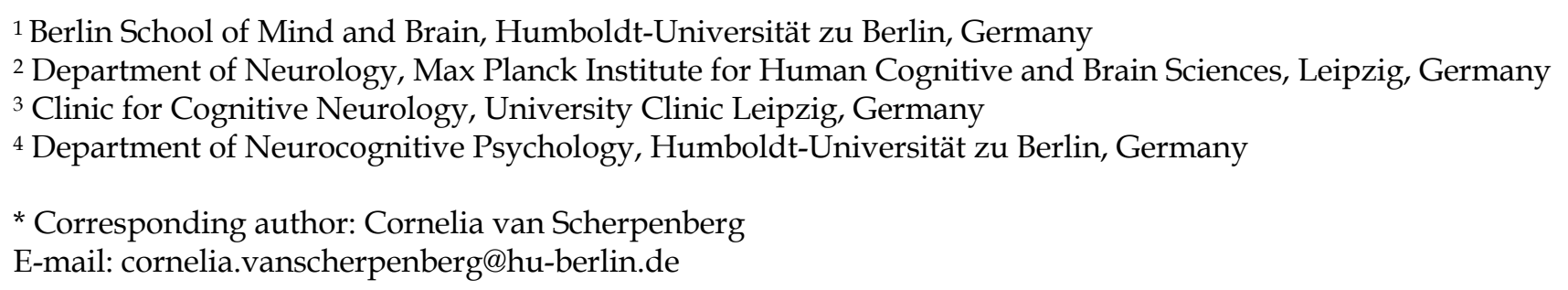

Unpublished manuscript, December 2020

\begin{abstract}
When we refer to an object or concept by its name, activation of semantic and categorical information is necessary to retrieve the correct lexical representation. While in neurotypical participants it is well established that semantic context can interfere with or facilitate lexical retrieval, these effects are much less studied in people with lesions to the language network and impairment at different steps of lexicalsemantic processing.

Here we applied a novel picture naming paradigm, where multiple categorically related and unrelated words were presented as distractors before a to-be-named target picture. Using eye tracking we investigated preferential fixation on the cohort members vs non-members. Thereby we could test whether explicit acknowledgement of the category is mandatory to induce semantic interference. We found that in contrast to neurotypical participants (van Scherpenberg, Abdel Rahman, \& Obrig, 2020), participants suffering from mild to moderate aphasia did not explicitly acknowledge the categorical relationship, but still showed a large interference effect of $\sim 35 \mathrm{~ms}$. On the contrary, preferential fixation on the categorically related cohort words correlated with higher non-verbal semantic abilities and integrity of the anterior temporal lobe (ATL). This highlights the role of supramodal semantics putatively housed in the ATL - for explicit semantic processing while semantic interference may be largely independent thereof. Our study adds further evidence to the observation that despite the implicit, automatic nature of semantic processing, it can influence naming speed and accuracy. This may be of particular importance to understand and intervene with linguistic deficits in people with aphasia.
\end{abstract}




\section{Introduction}

Impaired word retrieval is a hallmark of non-fluent language production in people with aphasia (PWA). Such impairment can surface through search behavior, slower and erroneous speech (e.g. in the form of semantic paraphasias, the substitution of a target word by a semantically related word (Schwartz, 2014)), or complete failure to produce certain words (anomia, Goodglass \& Wingfield, 1997; Kohn \& Goodglass, 1985). In psycholinguistic and neurolinguistic research, the analysis of specific deficits has shaped our understanding that word retrieval is a process consisting of several steps. In PWA each of these steps may be selectively impaired, leading to the observed patholinguistic patterns (Dell, Schwartz, Martin, Saffran, \& Gagnon, 1997; Levelt, Roelofs, \& Meyer, 1999). If an object (e.g., sheep) is to be named, it is assumed that through perceptual and conceptual processes, the target is recognized and its meaning is accessed. The process includes three major steps: (i) activation of semantic features that define the item, including category membership (ANIMAL), visual (e.g., IS WHITE) or functional (e.g., PRODUCES WOOL) features (e.g., Dell et al., 1997; Vigliocco, Vinson, Lewis, \& Garrett, 2004); (ii) retrieval of the object's name from the mental lexicon ("sheep"), and finally, (iii) access to the phonological representation of the target word [ji:p] followed by its articulatory realization.

Empirical research on both neurotypical and language-impaired populations has shown that accuracy and speed of naming varies if the respective processing levels are manipulated. For example, presenting the picture along with words phonologically or orthographically related to the target (e.g. sheep $p_{\text {PICTURE }}$ and sheet ${ }_{\text {WORD }}$ ) leads to facilitated and faster target naming (e.g., Abdel Rahman \& Melinger, 2008; Meyer \& Schriefers, 1991). This suggests phonological context to speed up the encoding of the phonological representation of the target word. In contrast, presenting context words which are semantically related to the target picture can have inhibitory effects on picture naming. In the picture-word-interference paradigm, a categorically related distractor word reduces picture naming latencies and increases error rates (e.g., Glaser \& Düngelhoff, 1984; Schriefers, Meyer, \& Levelt, 1990; Starreveld \& La Heij, 1996, 2017; Wheeldon \& Monsell, 1994). This effect, termed 'semantic interference effect', has been examined regarding the timing between distractor and target onset, the closeness of the semantic relation and has been shown for written and auditory presentation of the distractor. Beyond the many facets of manipulation, it stands as robust evidence for the assumption that semantic context influences language production (Bürki, Elbuy, Madec, \& Vasishth, 2020; de Zubicaray \& Piai, 2019).

Evidence on picture-word-interference in PWA is still sparse but has great potential to shed light on the impairment of the different processing steps involved in picture naming. In an auditory pictureword interference paradigm with one anomic patient, Wilshire, Keall, Stuart, and O'Donnell (2007) report significant semantic facilitation when target and distractor are presented simultaneously (Stimulus Onset Asynchrony, SOA = 0), while neurotypical participants show interference at this SOA. In the anomic patient, a trend towards semantic interference was only observed when the distractor was presented after the target picture onset (i.e. SOAs of +200 or $+400 \mathrm{~ms}$ ). Semantic interference effects are assumed to happen at the lexical selection stage through lexical competition, whereas priming or facilitation occur at the conceptual level (Bloem \& La Heij, 2003; Damian \& Bowers, 2003; Roelofs, 1992). Therefore the authors assume that the patient's semantic processing abilities are slowed down, prolonging the activation phase of the target's semantic representation. Thereby, at SOA $=0$ the distractor word would act on the semantic activation phase, not completed due to slowing. When semantic activation of the target is completed at later SOAs $(+200 /+400 \mathrm{~ms})$ the distractor would interfere with the delayed lexical retrieval step effecting a semantic interference effect. The example demonstrates that deviations from the pattern in neurotypical participants due to lesions to the language network impair naming at specific processing steps; this may allow for differentiation between these steps. Indeed, a later set of studies examining picture-word-interference in participants with aphasia describe different results. Hashimoto \& Thompson (2010) found significant semantic interference in reaction times at $\mathrm{SOAs}=$ 300 and 0 , with slightly bigger effect sizes but an otherwise similar pattern compared to agematched controls. Pino et al. (submitted) also report significant semantic interference for categorically related compared to unrelated distractor words in a group of 32 stroke patients (both in reaction times and errors, at SOA $=-100)$. Interference correlated 
with lesions to the inferior frontal cortex (IFG). Piai \& Knight (2017) likewise report significant semantic interference affecting reaction times and errors at $\mathrm{SOA}=0$ for a subgroup of participants with aphasia in their study. Interestingly the subgroup largely had lesions in the left lateraltemporal cortex. While the effect in reaction times was similar to that of controls, this patient subgroup showed a bigger interference effect in accuracy, with significantly larger error rates for semantically related compared to unrelated distractors. According to these latter studies, the pattern between neurotypical participants and those with aphasia thus seems to be comparable. Due to language production impairments in PWA, the effect shows more clearly in error rates, typically very low in neurotypical cohorts. In sum, studies in PWA support a differential impairment pattern depending on timing, semantic processing abilities and lesion site. While his prevents straightforward conclusions about semantic context effects in PWA, it offers a unique opportunity to study specific aspects of lexical retrieval.

In the current study we ask how a lesion to the left hemispheric language network alters two specific aspects of semantic context effects. To this end we invited participants with chronic but mild aphasia after left-hemispheric circumscribed chronic brain lesion to perform a novel multi-word interference paradigm previously established in neurotypical young speakers (van Scherpenberg et al., 2020). Naming abilities, assessed through voice onset time and errors, were complemented by a measure of semantic processing of the cohort using eye tracking. To account for the expected large interindividual differences we ran linear mixed models to analyse our results. Moreover, we correlated the variance of individual performance in the experiment with individual neuropsychological test scores and with individual lesion pattern.

The paradigm (introduced in van Scherpenberg et al., 2020) combines an assessment of 'semantic competence' using eye tracking with a measure of lexico-semantic processing through picture naming speed and accuracy in the presence of multiple categorically related distractor words. This allows for investigating semantic and lexical processes both separately, and in relation to each other. Using a circular display of eight distractor words, of which 3-5 belonged to one category while the

\footnotetext{
${ }^{1}$ We use this unspecific term to differentiate from Semantic
} Dementia, which is usually subsumed under the PPA remainder was semantically unrelated, we hypothesized that longer fixation on members of one semantic category will indicate 'semantic competence'. This assumption rests on our finding in neurotypical, young participants using the identical paradigm. However, in people suffering from Semantic Dementia it has been shown that with increasing loss of semantic knowledge participants spend more time fixating on unrelated foils compared to neurotypical participants with intact semantics (Faria, Race, Kim, \& Hillis, 2018; Seckin et al., 2016). The task used in that study was a word-to-object matching task, also called 'visual world paradigm' (Huettig, Rommers, \& Meyer, 2011). Hence impairment at the semantic processing level increases the difficulty in distinguishing between semantically related and unrelated items. In aphasia, evidence from the visual world eye tracking paradigm suggests that the participants were equally distracted from semantic competitors when having to point to the correct target picture, as were neurotypical controls (Yee, Blumstein, \& Sedivy, 2008, Experiment 1). This finding speaks for largely preserved conceptual activation of semantic relatives in typical $^{1}$ aphasia. Interestingly phonological onset similarity disclosed differences between PWA and controls: when the distractors presented in the picture set contained competitors whose semantic relative shared the same onset as the target, neurotypical controls were more likely to fixate on a picture of an object semantically related than on an onset competitor of the target (example: hammock TARGET $_{\text {and nail }}$ DISTRACTOR, via hammer). While PWA with a Wernicke-type aphasia showed a similar semantic onset competition effect, participants with Broca's aphasia did not (Yee et al., 2008, Experiment 3). This indicates that the dynamics of lexical activation are differentially impaired in different aphasia subtypes and are reflected in fixation preference.

The combined eye tracking and picture naming paradigm used in this study allows us to investigate in how far interference in naming depends on the processing of a semantic relationship. A common explanation for the semantic interference effect through distractor words is a trade-off between more short-lasting conceptual facilitation and longer lasting lexical competition (Bloem, van den Boogaard, \& La Heij, 2004). While a categorically related distractor may

(primary progressive aphasia) umbrella, but not limit 'typical aphasia' to the aetiology of stroke. 
prime the activation of the target through shared category nodes, when it comes to lexical selection, these co-activated lexical representations compete with each other, outweigh facilitation and therefore delay retrieval (e.g., Abdel Rahman \& Melinger, 2009, 2019; La Heij, Kuipers, \& Starreveld, 2006; Melinger \& Abdel Rahman, 2013; Wheeldon \& Monsell, 1994). In this competition account, interference is assumed to occur at the lexical selection step of the language production process. In our paradigm, fixation of the categorically related distractor words should pre-activate this category. Pre-activated potential lexical competitors should in turn hamper the selection of the lexical representation of the target picture resulting in a semantic interference effect commonly observed with single word distractors. However, if participants less efficiently distinguish between category members and non-members in the word set, they may not exhibit a strong semantic interference effect, because the category members did not activate a lexical cohort to induce competition. In the following, we will refer to this 'acknowledgement' of the semantic relationship between the distractor words, reflected by preferential fixation, as explicit semantic processing. Alternatively, if interference is preserved irrespective of the explicit processing of the semantic relationship, results would suggest that interference is not critically dependent on the acknowledgement of the semantic cohort, but that implicit, automatic semantic activation suffices. Evidence supporting this assumption comes from the continuous naming paradigm (Howard, Nickels, Coltheart, \& Cole-Virtue, 2006), where seemingly randomly presented pictures still induce interference during naming, even when unrelated pictures are named in between. Here, cumulative interference is induced despite the absence of explicit awareness of the categorical relationship. Measuring the fixation preference while reading the words therefore provides a measure of the dynamics of semantic content processing of distractor words.

For the clinical group who participated in our study, high-resolution structural MRIs were available allowing for lesion site delineation. We therefore included an exploratory analysis on lesion-symptom correlations to our investigations of the behavioural effects. Importantly, this allowed us to investigate lesion-symptom correlations for eye tracking measures such as fixations to semantic foils, i.e., the dynamics of semantic processing. The evidence from participants with Semantic Dementia points to an involvement of the left anterior temporal lobe in semantic competence, which is the primary atrophy site in this clinical group (Gorno-Tempini et al., 2011). The ATL has been considered a semantic hub necessary to gather and retrieve conceptual information about objects (e.g., Mesulam et al., 2009, 2013; Pobric, Jefferies, \& Lambon Ralph, 2007) and can therefore be hypothesized to be involved also in categorizing semantically related distractor words.

Only few studies so far have tried to relate the semantic interference effect in patients to specific lesion patterns and have yielded inconsistent results. Using voxel-based lesion-symptom mapping (VLSM), Pino et al. (submitted) were able to relate the effect to lesions in the inferior frontal gyrus. More precisely, lesions in the IFG correlated with an increased semantic interference effect in naming latencies. Additionally, overall latencies in the naming task were slowed down in participants with lesions in the middle temporal gyrus (MTG) suggesting an involvement of this area in the lexical selection process. This is in line with previous findings that lesions in MTG influence picture naming in patients with aphasia (Piai \& Knight (2017). Piai and Knight report significant semantic interference in a picture-word-interference task for patients with primary lesions in the left lateraltemporal cortex (primarily in the superior temporal gyrus (STG) and MTG). On the contrary patients with lesions in the left prefrontal cortex (middle frontal gyrus (MFG) and IFG) did not exhibit and interference effect. The exact role of the left PFC and IFG in particular during the language production process is still elusive (see also Mirman et al., 2015; Riès, Karzmark, Navarrete, Knight, \& Dronkers, 2015). Recent reviews by de Zubicaray \& Piai (2019) and Nozari (2020) confirm that even taking into account neuroimaging studies, there is not yet a consensus on how exactly brain regions affording language production process are involved in the semantic interference effect. Moreover the paradigm applied here deviates in several aspects from the classical picture-word-interference paradigm. Therefor our hypotheses concerning the VLSM analysis remain tentative. As a starting point we assume a correlation of potential effects in naming latencies with lesions in more frontal areas in the left PFC and more temporal areas in the left lateral-temporal cortex. Based on the literature regarding semantic dementia lesions to the left ATL 
can be hypothesized to correlate with eye tracking patterns reflecting impairment of overall semantic competence.

\section{Methods}

\section{Participants}

32 participants with chronic lesions in the left hemispheric language network, aged 17-73 years (mean $=53, s d=11.5,10$ women), participated in this study in return for monetary compensation of $€ 9$ per hour. They were selected from a database of the Clinic for Cognitive Neurology (University Hospital Leipzig) and the Max Planck Institute for Human Cognitive and Brain Sciences (MPI-CBS). Exclusion criteria for participation were additional right-hemispheric lesions, severe overall cognitive impairment, severe apraxia of speech or reading impairments. All participants had normal or corrected to normal vision.

All participants were diagnosed with aphasia or residual aphasia at the time of inclusion, based on the standard German assessment battery (Aachener Aphasie Test, AAT, Huber, Poeck, Weniger, \& Willmes (1983)). Of the 32 participants 4 participants were excluded from the final sample due to too many invalid eye tracking samples, or errors in voice recording. In all remaining 28 participants structural brain imaging was available allowing for lesion delineation. 23 participants had a high-resolution structural MRI acquired at the MPI-CBS, in 5 participants clinically motivated MRIs with lower slice resolution were used (for details see section 2.4. below). The overlay of all participants is shown in Figure 1. Note that besides the temporal lobe the IFG is covered be the lesion overlap.

Participants underwent extensive cognitive and language-related assessments. A detailed summary of each participant's demographic and clinical information as well as their cognitive and language abilities is shown in Table 1.

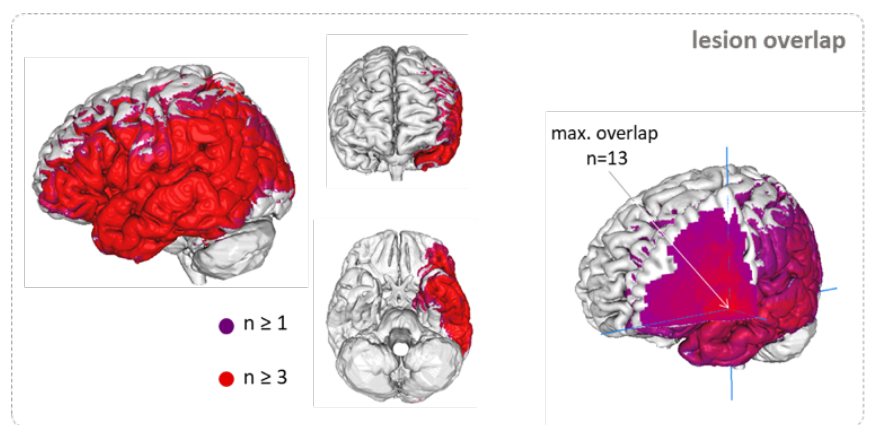

Figure 1: Overlay of all 28 patients in whom the analyses were performed. Note that the 'field of view', that is a lesion overlap in 3 and more participants, covers the temporal lobe, the temporo-parietal junction and the inferior frontal gyrus. Maximal overlap $(n=13)$ is located in the insular region, which is seen in all studies dominated by stroke lesions.

Experimental procedures were approved by the Institutional Review Board of the University of Leipzig, Germany, in accordance with the Declaration of Helsinki, and written informed consent was obtained from all participants (Ethical approval to AZ 144/18-ek, Ethics Committee University Leipzig). 


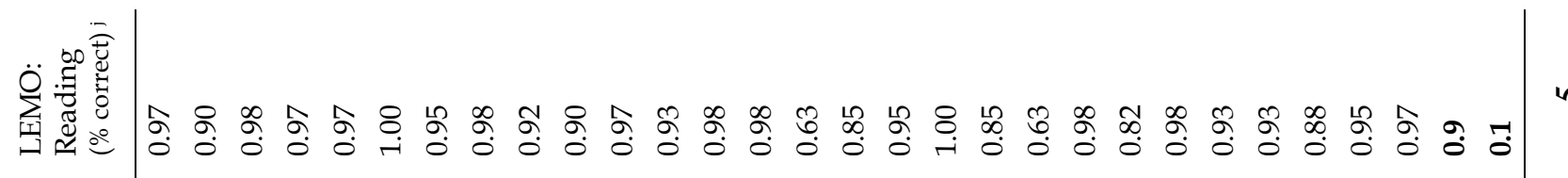

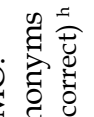

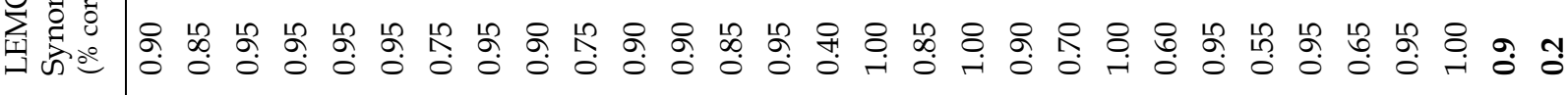

它

ఏ̆

$\stackrel{\dot{0}}{\stackrel{0}{\leftrightarrows}}$

$\overline{0}$
$\vdots$
3

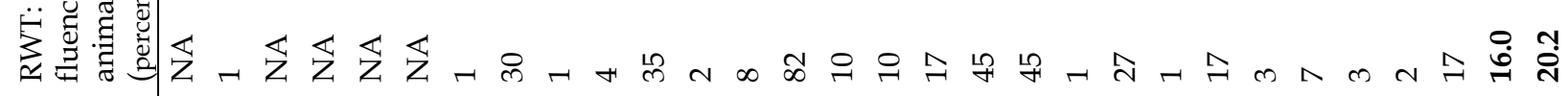

:

흐

ธิ

.0

巳

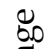

荧

$\frac{\pi}{\square}$

.

50

용

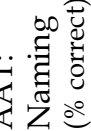

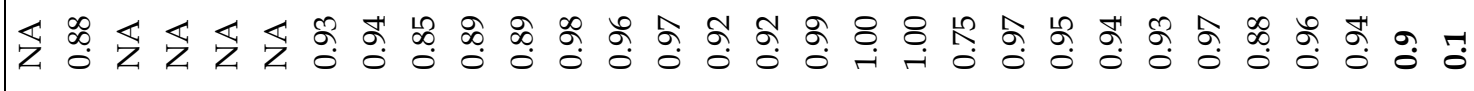

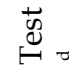

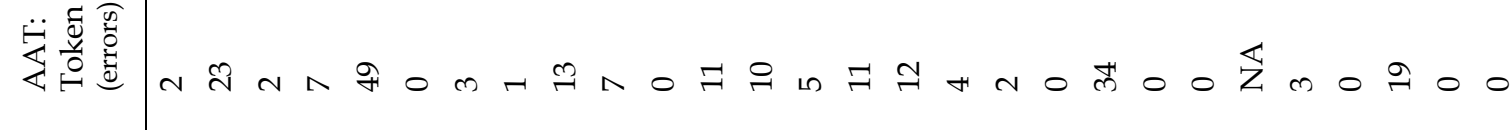

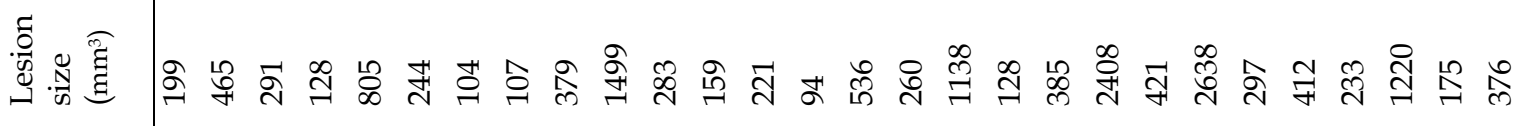

ज्ञ

褐 密

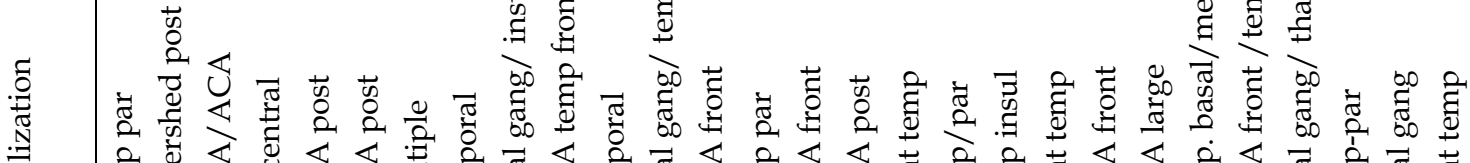

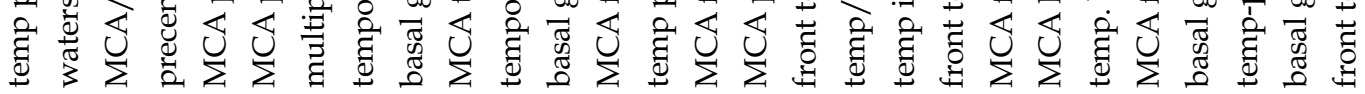

एँ

g

:ี

|

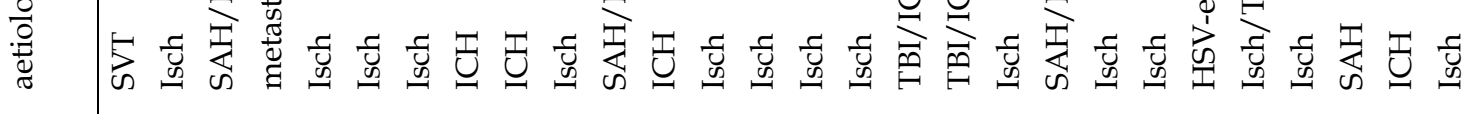

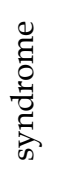

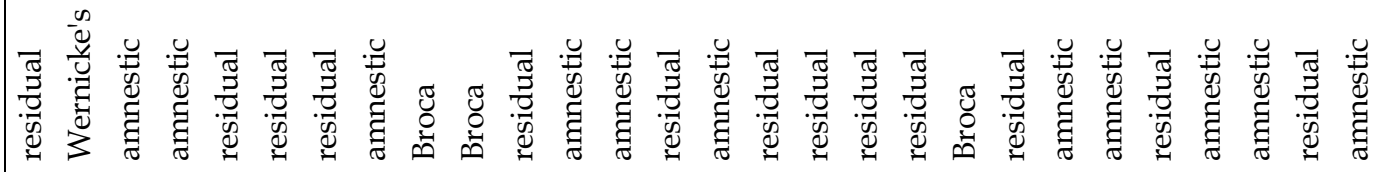

ن.

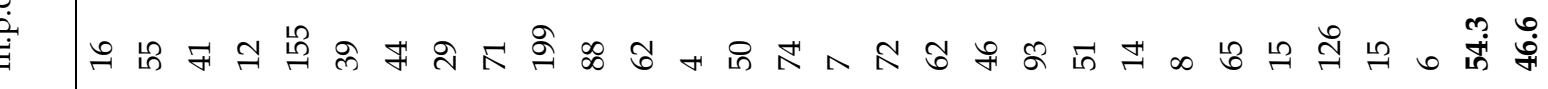

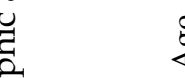

俘 F

Uัँ

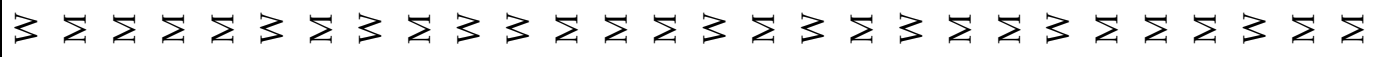

苞 


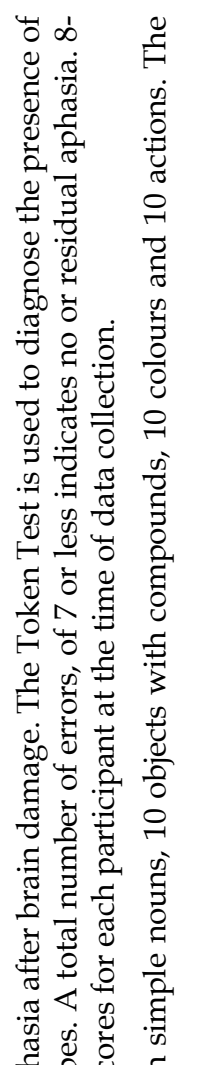

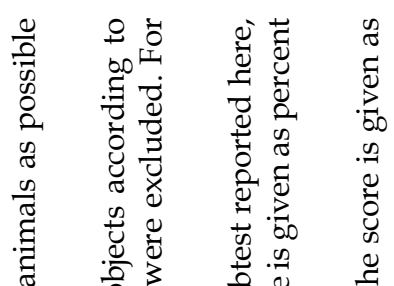

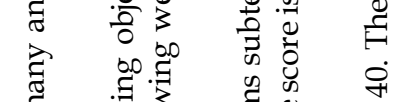

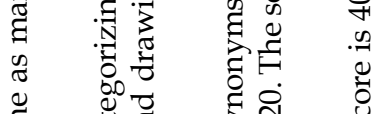

范芩

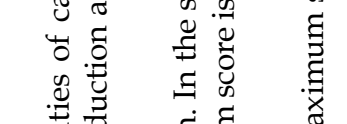

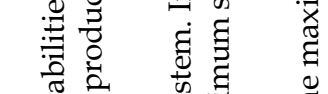

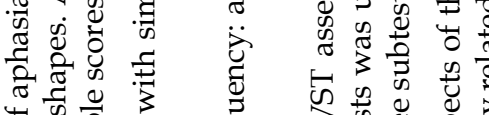

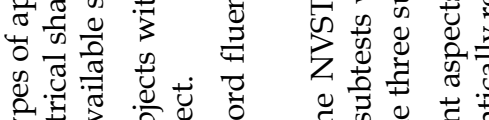

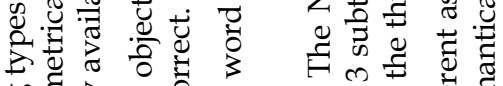

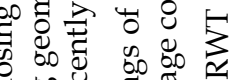

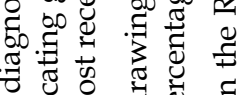

它苍 की

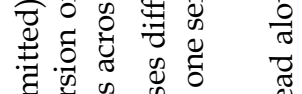

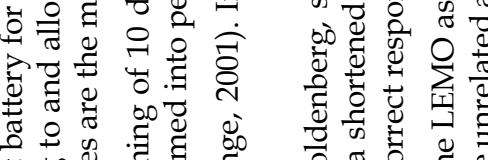

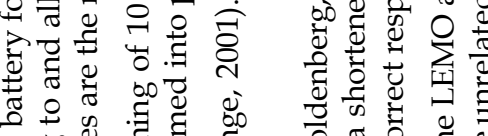

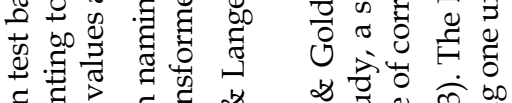

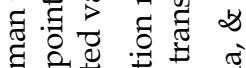

ते

Е

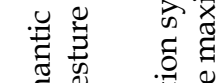

एक

के

है

즌.

$\frac{\pi}{5}$

$\frac{\sqrt{3}}{\frac{7}{9}}$

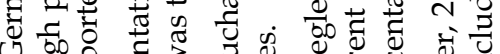

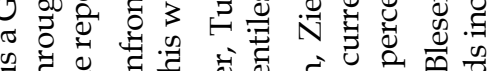

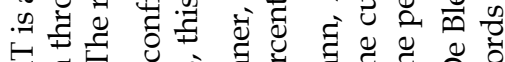

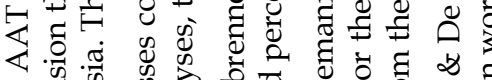

券

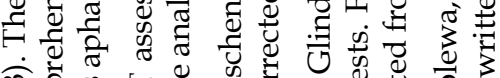

बक

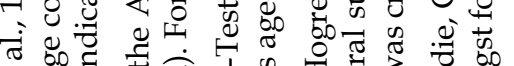

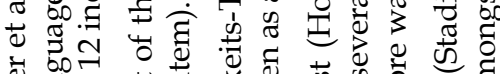

पँ⿱

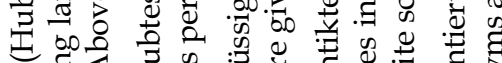

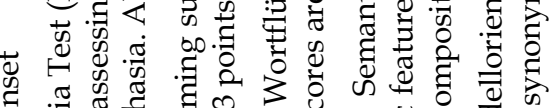

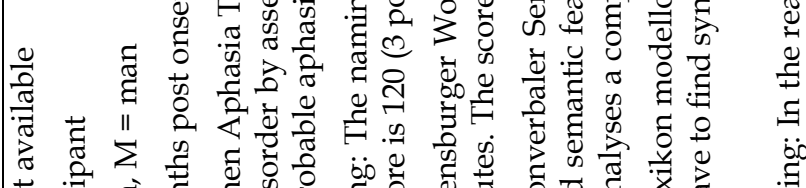

苛 全

花 ह

$Z$ Z $\|$ है एँ

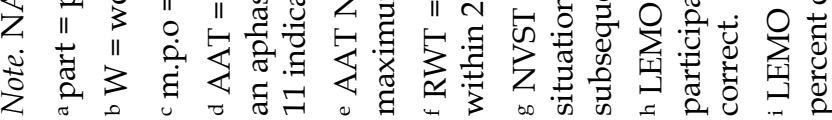




\section{Materials}

We used a variation of the picture-word interference approach, which is described in detail in van Scherpenberg et al. (2020). In this paradigm, instead of one, 8 distractor words are presented simultaneously in each trial, in the shape of a circle, followed by the picture to be named. Out of these sets of 8 words a varying number $(3,4$ or 5$)$ belong to one semantic category, while the remaining words each stem from a different, unrelated category. The target pictures are either part of this semantic category, or entirely unrelated to any of the distractor words. The material was constructed using 7 semantic subcategories with 6 members each, resulting in a total of 42 items. See Table A1 (Appendix) for an overview of the stimuli.

\section{Apparatus}

The stimuli were presented using the Psychophysics Toolbox extension (Brainard, 1997) for MATLAB (2017a, MathWorks, Inc.) on a Lenovo Thinkpad T420 laptop (14" monitor, $1600 \times 900$ pixels resolution). The words were presented in white Arial font, size 40, on a black screen, and the pictures were scaled to $5.8 \times 5.8 \mathrm{~cm}$ (300x300 pixels, $5.5^{\circ}$ of visual angle at a distance of $60 \mathrm{~cm}$ between the viewer's eyes and the screen). Eye movements were recorded from both eyes using a Tobii X2-60 eye tracker with a 60 Hertz sampling rate. Voice responses were recorded using a Blue Yeti USB microphone.

\section{Design and Procedure}

The variation of the number of related words in the distractor set results in a $2 \times 3$ design with picture TYPE (related vs unrelated) and SIZE of lexical cohort $(3,4$ or 5$)$ as within-participants factors. 12 randomized lists were created with the constraints that target pictures were separated by a minimum of two other items and that each target appeared once with a related and once with an unrelated distractor set in each block. Across each list, the participants therefore named each item six times. The lists were randomly assigned to the participants, by which each list was repeated a maximum of three times.

Each experimental session started with an instruction of the experimental procedure to which the participants consented. They were then seated in a dimly lit, sound-proof room in front of the laptop and eye tracker with a distance of approximately $60 \mathrm{~cm}$ to the screen. A chin rest was used to minimize head movements and improve eye-tracking data quality.

To familiarize the participants with the materials, each picture was presented centered on the screen with its name written underneath. In a self-paced manner, the participants named one picture after the other, and this procedure was repeated if items were not correctly named after the first familiarization (this applied only to one participant). At the start of the experimental session, the eye tracker was calibrated according to a 5-point calibration procedure, followed by three practice trials, after which any remaining questions were addressed by the experimenter.

The experimental trials were split up in three blocks with 84 trials each, in between which participants were able to take a break. Note that presentation times were increased slightly compared to the original procedure described in van Scherpenberg et al. (2020) (8s instead of $6 \mathrm{~s}$ for the words, and $4 \mathrm{~s}$ instead of $2 \mathrm{~s}$ for the pictures). This accounts for additional processing costs in participants with aphasia. At the start of each trial a fixation cross was presented in the center of the screen $(0.5 \mathrm{~s})$, directly followed by the set of the eight distractor words presented in a circle around the center of the screen for $8 \mathrm{~s}$ (see Figure 2 for a typical trial procedure). Participants were instructed to inspect the word set freely but were told that a minimum of three of the eight words were related to each other. During this part of the trial, participants' eye movements were recorded by a Tobii X2-60 eye tracker. Directly after the $8 \mathrm{~s}$, the distractor words disappeared, and the target picture was presented for $4 \mathrm{~s}$. Participants were instructed to name the picture as quickly and accurately as possible and their response was recorded. After an inter-trial interval of $0.5 \mathrm{~s}$, the next trial started automatically. Each trial thus lasted for $13 \mathrm{~s}$, resulting in a total experiment time of around 54 minutes, not including breaks. 


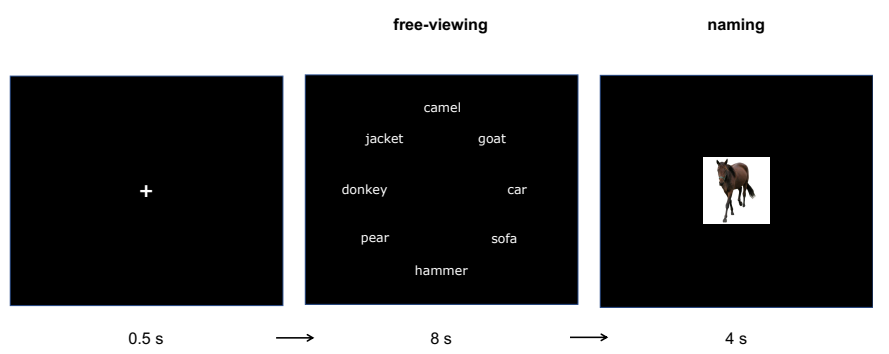

Figure 2: Exemplary procedure of a trial in which the word set contains a lexical cohort of three items from the semantic category "hoofed animals" (SET SIZE $=3$ ). The target picture is part of the same semantic category (PICTURE TYPE = related). In the actual experiment, the words were presented in German.

\section{Lesion-Behaviour Correlations}

For all participants entering the analysis $(n=28)$, structural imaging was available. 23 scans were performed at in-house MRI scanners (3T Siemens MRI system Trio ${ }^{\circledR}$ or Verio ${ }^{\circledR}$ system, Siemens Medical Systems, Erlangen, Germany) including 3D T1-weighted- (1 $\mathrm{mm}^{3}$ isovoxel), and FLAIRimages. In 5 participants MRIs from clinically motivated imaging were available, with partially lower resolution (3-5 $\mathrm{mm}$ slice thickness, including FLAIR or TIRM and T1 images). Manual lesion delineation was performed by an experienced neurologist (H.O.) primarily based on T1 images respecting the (lower resolution) FLAIR/ TIRM images. This was done using MRIcron (Rorden and Brett, 2000). All images were then transformed into standard stereotactic space (MNI) @1 $\mathrm{mm}^{3}$ using SPM12 (www.fil.ion.ucl.ac.uk/spm) and the "clinical toolbox" (nitrc.org/projects/clinicaltbx/). The unified segmentation approach was applied (Ashburner and Friston, 2005) and estimation of normalization parameters was restricted to healthy tissue using predefined lesion masks (Brett et al., 2001).

Lesion-behavior correlations were performed along the principles of multivariate lesionsymptom mapping based on support-vectorregression (Zhang, Kimberg, Coslett, Schwartz, \& Wang, 2014). The publicly available software used here is based on this approach (SVR-LSM toolbox running in a MATLAB environment as published in DeMarco \& Turkeltaub, (2018), https://github.com/dmirman-zz/SVR-LSM). Multivariate approaches have the advantage that they take into account inter-voxel correlations. Estimating lesion-symptom maps for all voxels simultaneously lesion mislocalization is attenuated while sensitivity to nonlinear relationships is enhanced (Zhang et al., 2014). The package used (SVR-LSM toolbox) provides several methods for controlling for lesion size. This is a central issue in all lesion-behavior approaches; most intuitively it means that a behavioral difference between participants lesioned vs. non-lesioned in a specific voxel is more likely to be due to a lesion elsewhere, if the overall lesion of a participant is larger². Here we chose lesion volume correction of both the behavioral scores and the lesion maps, as is recommended by (DeMarco \& Turkeltaub, 2018). Only voxels in which 3 and more participants showed lesions were included. The parameters analyzed were voice-onset time as recorded by the voice key (VOT), fixation time as monitored by the eye tracker (FIX) and the performance in two of the clinical tests in percent correct: (i) non-verbal semantic assessment, NVST (Hogrefe et al., submitted) and (ii) decision on visually presented synonyms with distractors, from the LEMOBattery (Stadie et al., 2013). Error Rates were low and were not analyzed (in analogy to the LMMs regarding the behavioral analyses).

To infer statistical significance the approach first assesses voxel-wise statistical significance by permutation testing. In the present study 2000 permutations were performed and only SVR- $\beta$ values with a $p<.005$ were regarded further. SVRLSM considers all voxels simultaneously in a single model, however, to further reduce the multiple comparison issue a second step is based on familywise error rate (with $p<.05$ ) for the clusterextent threshold determined from the permutations (DeMarco \& Turkeltaub, 2018).

\section{Analysis}

\section{Picture Naming}

Voice onset times. For the correct trials, voice onsets were determined at the start of each word, excluding stuttering, "uhms", or search behavior before a correct response. The voice onset times (VOTs) were detected using the Chronset algorithm, (Roux, Armstrong, \& Carreiras, 2017), and checked manually using Praat (Boersma \& Weenink, 2018). These were considered as the overt response, i.e., the reaction times.

\footnotetext{
${ }^{2}$ Vice versa: if a participant showed a lesion in one single voxel only, the performance deficit could be precisely ascribed to this voxel.
} 
Trials in which the voice recording was missing due to technical errors of the microphone (e.g. missing recordings or white noise) where discarded from all analyses $(2.9 \%, \mathrm{n}=198$ from all 28 participants).

Responses were considered incorrect and treated as errors when participants did not respond at all, responded falsely, or made false starts even when they consecutively produced a correct response $(7.5 \%, \mathrm{n}=510)$. In total, $10.4 \%(\mathrm{n}=708)$ of all trials were classified as errors; these trials were excluded from the VOT analysis.

Error coding. All erroneous responses were classified according to whether they were (1) noresponses, (2) semantic errors (e.g., semantic coordinates or superordinates), or (3) other errors (e.g., phonological errors, visual errors (for example moon $\rightarrow$ "banana"), unrelated responses).

\section{Eye Tracking Data}

From the raw data samples fixations and saccades were detected using the GazePath algorithm (van Renswoude et al., 2018) on the mean $x$ - and ycoordinates of the left and right eye. Areas of Interest (AoI) were defined as rectangles of 270 x170 pixels around each word in the circular word set. To correct fixations which were distorted due to head movements, we calculated the minimum euclidean distance between each data point in the fixation data frame and the 8 AoIs. This value was then used to adjust the drift of each distorted data point towards the position of the respective word in the word set.

Observations where GazePath failed to detect any fixations were excluded from analysis $(\mathrm{n}=3344$, $5.9 \%$ ). Combining data loss from VOT and eye tracking data, around 15\% were not available for the consecutive statistical data analysis.

\section{Statistical Analysis}

All statistical analyses were performed using $\mathrm{R}$ version 3.6.1 ( $\mathrm{R}$ Core Team, 2016). Linear mixed effect (LME) models were run with random slopes for subjects and items, using the lme4 package in $\mathrm{R}$ for linear mixed models (version 1.1-21; Bates, Maechler, Bolker, \& Walker, 2015), and $p$ values were determined using the package lmerTest (Kuznetsova, Brockhoff, \& Haubo, 2016). All code and anonymized data can be downloaded here: https://osf.io/ezcgk/.

\section{Results}

\section{Voice onset times and naming errors}

The mean VOT for the group across naming conditions is provided in Table 2 and illustrated in Figure 3. Overall, naming of semantically related pictures was $34 \mathrm{~ms}$ slower compared to naming of unrelated pictures, and descriptively, this interference effect was strongest for the condition with 4 distractor words (42 ms). On average, participants made 11 errors for related pictures and 10 errors for unrelated pictures. Given a total number of 126 trials per picture type condition, these error rates are quite low (around $8 \%$ of all trials). Considering that across set sizes the error rates were even lower, and that descriptively, the differences between conditions were minor, we did not analyze errors further statistically.

Table 2: Mean VOTs in milliseconds and Standard Error of the Means for each naming condition.

\begin{tabular}{lllllllll} 
Distractor set size & 3 & \multicolumn{2}{c}{4} & \multicolumn{2}{c}{5} & \multicolumn{3}{c}{ total } \\
\hline Picture type & rel & unrel & rel & unrel & rel & unrel & rel & unrel \\
Mean VOTs in ms & 1333 & 1302 & 1336 & 1294 & 1327 & 1297 & 1332 & 1298 \\
SE & 16.07 & 15.25 & 15.65 & 14.78 & 16.19 & 15.24 & 11.90 & 11.25 \\
Interference & 30.85 & & 42.00 & & 29.41 & & 34.11 & \\
Mean no. of Errors & 4.74 & 5.06 & 3.86 & 3.86 & 4.40 & 4.05 & 10.96 & 10.32
\end{tabular}

Note . rel $=$ related, unrel $=$ unrelated, $\mathrm{SE}=$ Standard Error of the Mean. Values are adjusted for within-participant designs following Morey (2008).

The effects were confirmed in a GLMM with a fully specified random structure (main effects and interaction term for picture TYPE and set SIZE for both Subjects and Stimuli) without correlation parameters. Sliding difference contrasts were used to code the pairwise comparisons of picture type (related vs. unrelated) and set size (5 vs 3 and 4 vs 3 distractor words) directly within the model.

The analysis revealed a significant main effect of picture type, indicating that related pictures were named significantly slower than unrelated pictures (estimate $=34.71, \mathrm{SE}=15.08, \mathrm{t}=2.3, \mathrm{p}=0.021$ ). The main effect of set size was non-significant (4 vs. 3 distractor words: estimate $=-4.96, \mathrm{SE}=13.61, \mathrm{t}=$ $0.36, p=0.715 ; 5$ vs 3 distractor words: estimate $=$ $6.16, \mathrm{SE}=15.8, \mathrm{t}=-0.39, \mathrm{p}=0.697)$. Importantly, the interactions between picture type and set sizes were non-significant as well (for 4 vs 3 and 5 vs 3 distractor words both ts $<0.54, \mathrm{p}>0.587$ ). This was confirmed by a nested model, where the effects of picture type were nested under the levels of set 
size. The results reveal a statistically significant interference effect at all set sizes (3 distractor words: estimate $=36.25, \mathrm{SE}=5.2, \mathrm{t}=6.97, \mathrm{p}<0.001$; 4 distractor words: estimate $=37.86, \mathrm{SE}=7.28, \mathrm{t}=$ 5.2, $\mathrm{p}<0.001$; 5 distractor words: estimate $=28.88$, $\mathrm{SE}=6.54, \mathrm{t}=4.42, \mathrm{p}<0.001)$. This means that, contrary to our predictions, additional distractor words did not significantly affect naming latencies. These results are summarized in Figure 3. For comparison, we provide results by neurotypical participants $(n=24)$ described in van Scherpenberg et al. (2020) in the same plot.

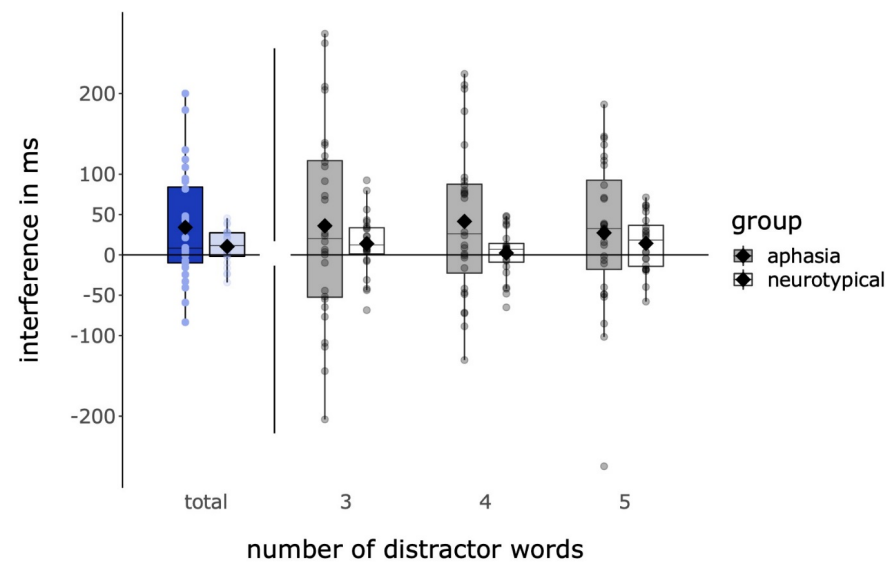

Figure 3: Interference effect in total and across number of distractor words for participants with aphasia (darker shades) and neurotypical participants (lighter shades, $n=24$, see van Scherpenberg et al. 2020 for details). Boxplots show mean, median, upper and lower quartiles and range. Dots represent individual means. For numerical values see Table 2 .

Establishing this novel paradigm in young, neurotypical participants (van Scherpenberg et al., 2020) we found an item repetition effect. Item repetition had an effect on naming latencies and the semantic interference effect, reducing both significantly across repetitions. We therefore ran another model for the clinical population in the current study, including repetition as a covariate (mean-centered and standardized) in interaction with picture type. The fully specified random structure was supported and reached convergence. This analysis revealed main effects of picture type (estimate $=39.75, \mathrm{SE}=15.19, \mathrm{t}=2.62, \mathrm{p}=0.009$ ) and repetition (estimate $=-65.68, \mathrm{SE}=9.94, \mathrm{t}=-6.61, \mathrm{p}$ $<0.001)$. In contrast to the neurotypical population, however, results in the clinical population show no interaction between picture repetition and picture type (estimate $=8.02 . \mathrm{SE}=15,17, \mathrm{t}=0.53, \mathrm{p}=0.597$ ). This indicates that although naming latencies decreased linearly by $\sim 66 \mathrm{~ms}$ on average across repetitions, the semantic interference effect remained stable in the clinical population as illustrated in Figure 4, again including the comparison to the neurotypical population.

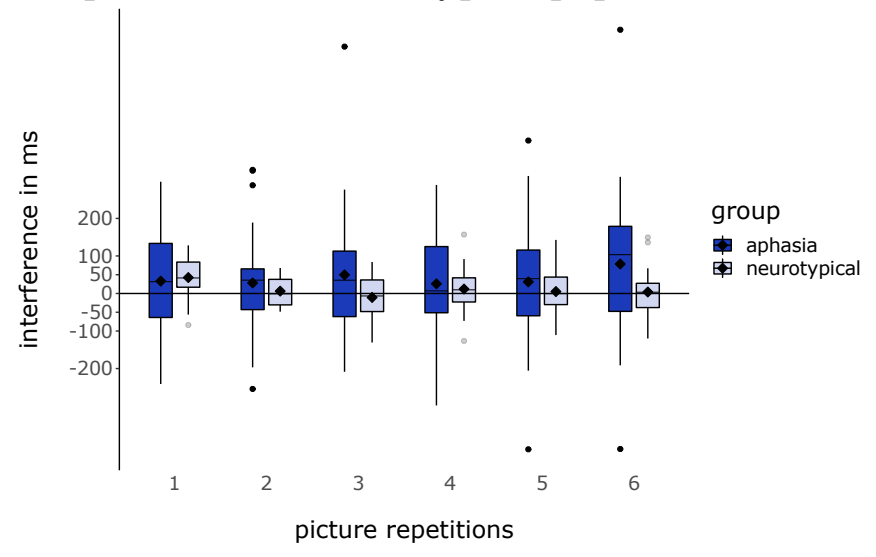

Figure 4: Interference effect across picture repetitions for participants with aphasia (darker shades) and neurotypical participants (lighter shades, $\mathrm{n}=24$ see van Scherpenberg et al. 2020 for details). Boxplots show mean, median, upper and lower quartiles and range. Dots represent individual means.

\section{Fixation durations}

Prior to the naming task, participants viewed the distractor words arranged in a circle and fixations on these words were analyzed separately. Note that in each trial, 3, 4 or 5 words were members of a semantic category while the remaining $(5,4$ or 3 respectively) were non-members. To evaluate statistically the difference in fixation durations between the category members vs. non-members, we ran a generalized linear mixed model with fixation durations as the dependent variable and word type (member vs. non-member) and set size $(3,4$ or 5$)$ as fixed effects. All fixed effects were coded with sliding difference contrasts. Again, we accounted for by-subject and by-stimulus random slopes, and the final converging model included a fully specified random structure, without correlation parameters and one contrast of the factor set size. None of the contrasts were significant, indicating no significant difference between fixation durations on category members vs. non-members, independent of the number of categorically related words (all ts $<0.874$, all ps $>$ 0.382 , see Figure 5 and Figure B1 for details). As described in van Scherpenberg et al. (2020) and illustrated in Figure 5, this finding is clearly different from the pattern we observed with neurotypical participants, who fixated longer on category members independent of set size. 


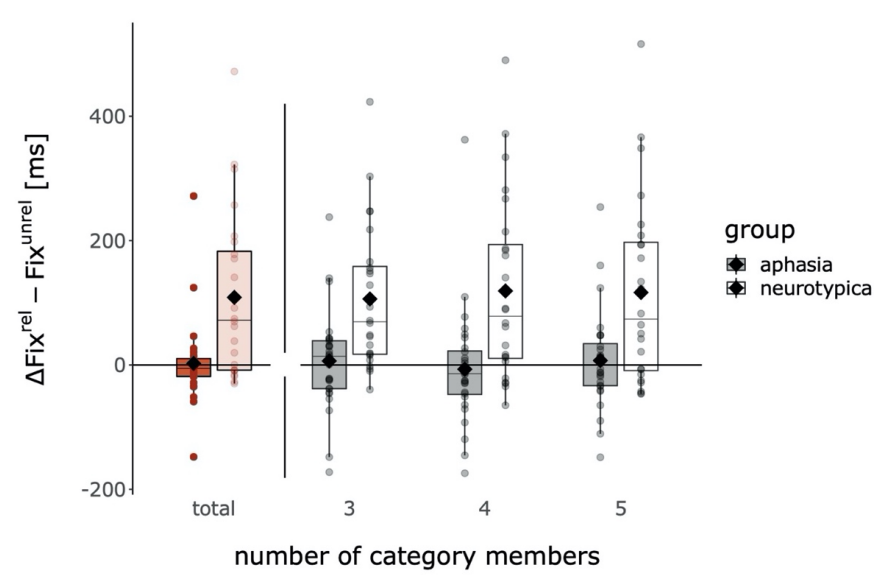

Figure 5: Fixation durations for each word as part of the distractor word set for participants with aphasia (darker shades) and neurotypical participants (lighter shades). Boxplots show mean, median, upper and lower quartiles and range. Dots represent individual means.

Subsequently, we investigated whether fixation durations on category members had an effect on naming latencies, in interaction with picture type. We therefore added fixation durations as a covariate (mean-centered and scaled) to a generalized linear mixed model with VOT as dependent variable and picture type as fixed effect. The model with a fully specified random structure without correlation parameters converged.

The analysis revealed no influence of fixation durations on naming latencies (estimate $=2.88$, SE $=10.33, \mathrm{t}=0.28, \mathrm{p}=0.781)$ and no interaction with picture type (estimate $=10.04, \mathrm{SE}=15.81, \mathrm{t}=0.64$, $p=0.525)$. The main effect of picture type remained significant (estimate $=31.27, \mathrm{SE}=15.47, \mathrm{t}=2.02, \mathrm{p}$ $=0.043$ ).

\section{Correlations with clinical linguistic measures}

Finally, we correlated a selection of the clinical linguistic measures with our experimental effects. The linguistic measures (see Table 1) included reading abilities (LEMO Reading), synonym judgments (LEMO synonyms), general naming abilities (AAT Naming), semantic word fluency (RWT: animals) and non-verbal semantic abilities (NVST). We were particularly interested in how these measures might be related to the semantic interference effect, or the difference in fixation durations between category members and nonmembers. These individual effect sizes were taken from the estimated Subject coefficients of the linear mixed models described above to account for interindividual or item-based variation. The semantic interference effect did not correlate with any of the test scores. However, performance in the NVST correlated with the differences in fixation durations to category members vs. non-members (rho $=0.463, \mathrm{p}=0.013$ ): The better participants scored in the NVST, the more they preferentially fixated on categorically related words, indicating acknowledgment of the semantic category. The (Spearman rank) correlations are summarized in Table C1 (Appendix).

\section{Lesion-behavior correlations}

In our exploratory lesion-behavior analysis we used mean individual voice-onset times (VOT) and fixation times (FIX) during the exploration of the 8 distractor words. For both parameters we analyzed the respective value for the related and the unrelated conditions and their difference. Moreover, we correlated individual lesions with individual scores in the clinical tests.

For VOT the behavioral score was the mean response time after picture onset with VOTrel if the picture belonged to the same category as the semantic category in the distractor word set; and VOTunr for pictures unrelated to the category. For the analyses the respective other parameter was entered as a covariate in the SVR-LSM model (i.e. VOTrel as a covariate for the analysis of VOTunr and vice versa). An additional analysis was performed with the mean difference between related and unrelated conditions ( $\Delta$ (VOTrel-VOTunr)). In this case VOTmean was introduced as a covariate in the SVR-LSM. For fixation time the same values were calculated (i.e. FIXrel, FIXunr and $\Delta$ (FIXrel-FIXunr)). Regarding the interpretation of the results it is relevant to consider the assumptions of the SVRLSM model. While for overall VOT it is intuitive that larger values are expected as a sequel of a lesion in a relevant brain area the strength of interference effect may result in a seemingly paradoxical behavior. That means that a lesion may attenuate the interference effect, thereby resulting in relatively shorter VOT compared to a participant without a lesion. Therefore results listed in Table 3 and illustrated in Figure 6 and Figure 7 regard lesion sites which correlate with a deviation from the expected behavior (which has been documented in the neurotypical young control group). The clusters listed and illustrated did not survive the more conservative cluster-based correction for multiple comparisons. Therefore, results must be interpreted with caution. 
Table 3: Results of the SVR-LSM. The largest clusters for the respective parameters are listed. Note that in the clusters each voxel passed the $\mathrm{p}<0.005$ threshold, while none of the clusters survived more conservative cluster-based correction. The table provides the cluster size in $\mathrm{mm}^{3}$ and as the diameter of a sphere corresponding to the volume (Dia). pmax denotes the maximal statistical threshold reached in the cluster. MNI coordinates and regions according to 3 different atlases are provided (Harvard-Oxford atlas, Automated anatomical labelling atlas, Brodman atlas; n.d. indicates not defined in the atlas). The direction of the arrow in the first column indicates the direction in which a lesion would modulate the parameter, i.e. $\downarrow$ : lesion will decrease the numerical value and $\uparrow$ : lesion will increase the numerical value

\begin{tabular}{|c|c|c|c|c|c|c|c|c|c|}
\hline & \multirow{2}{*}{$\begin{array}{c}\text { size } \\
\text { mm }^{3}\end{array}$} & \multirow{2}{*}{$\begin{array}{l}\text { Dia } \\
\text { mm }\end{array}$} & \multirow[b]{2}{*}{$p^{\max }$} & \multicolumn{3}{|c|}{ MNI of peak } & \multirow[b]{2}{*}{ Harvard } & \multirow[b]{2}{*}{ aal } & \multirow[b]{2}{*}{ Brod } \\
\hline & & & & $\mathbf{x}$ & $\mathbf{y}$ & $\mathbf{z}$ & & & \\
\hline \multicolumn{10}{|l|}{ VOT } \\
\hline \multirow[t]{2}{*}{ mean $\uparrow$} & 273 & 4.0 & 0.001 & -45 & -55 & 0 & MTGtemp-occ & MTG & 37 \\
\hline & 243 & 3.9 & 0.002 & -27 & -51 & 26 & n.d. & n.d. & n.d. \\
\hline \multirow[t]{2}{*}{$\Delta$ (rel-unr) $\downarrow$} & 218 & 3.7 & 0.001 & -47 & -55 & 3 & MTGtemp-occ & MTG & 37 \\
\hline & 133 & 3.2 & 0.001 & -41 & -6 & -12 & pl. polare & STG & n.d. \\
\hline rel $\downarrow$ & 222 & 3.8 & $<0.001$ & -41 & -6 & -12 & pl. polare & STG & n.d. \\
\hline unr $\uparrow$ & 407 & 4.6 & 0.002 & -47 & -54 & -1 & MTGtemp-occ & MTG & 37 \\
\hline \multicolumn{10}{|l|}{ FIX } \\
\hline \multirow[t]{2}{*}{ mean $\downarrow$} & 957 & 6.1 & 0.001 & -48 & 1 & -41 & ITGant & ITG & 20 \\
\hline & 301 & 4.2 & 0.001 & -67 & -40 & -20 & ITGant & ITG & 20 \\
\hline$\Delta$ (rel-unr $) \downarrow$ & 620 & 5.3 & 0.001 & -43 & -1 & -43 & ITGant & ITG & 20 \\
\hline rel $\downarrow$ & 2316 & 8.2 & 0.002 & -34 & 3 & -42 & temp pole & temp pole mid & 36 \\
\hline unr $\uparrow$ & 81 & 2.7 & 0.004 & -34 & 3 & -42 & temp pole & temp pole mid & 36 \\
\hline \multicolumn{10}{|l|}{ CLINICAL } \\
\hline NVST \% $\downarrow$ & 4643 & 10.3 & $<0.001$ & -46 & -9 & -46 & ITGant & ITG & 20 \\
\hline SYN\% $\downarrow \downarrow$ & 103 & 2.9 & $<0.001$ & -48 & 17 & -13 & temp pole & temp pole sup & 38 \\
\hline
\end{tabular}


As illustrated in Figure 6 lesions in a cluster in the anterior inferior temporal gyrus reduced mean fixation time during the presentation of the distractor words, while a cluster in the posterior portion of the MTG increased VOT signaling slower naming. The second cluster corresponding to VOT increases is located in the subcortical white matter.

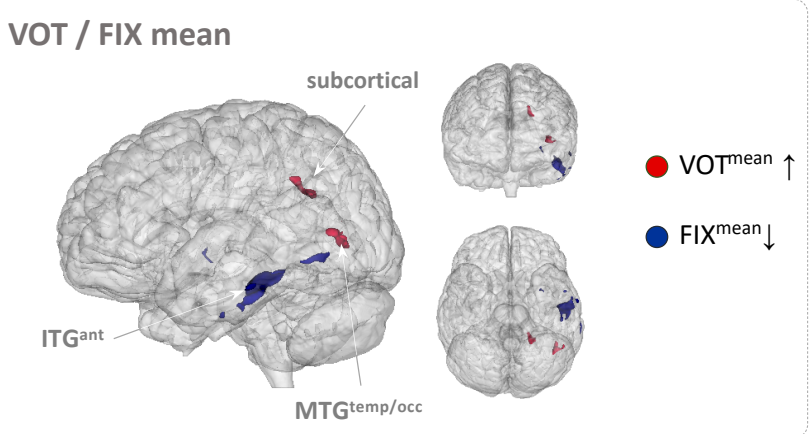

Figure 6: Clusters (uncorrected) corresponding to shorter fixation during the presentation of the distractor words (FIXmean $\downarrow$, blue) and longer naming latencies (VOTmean $\uparrow$, red). Specifications of the clusters are provided in Table 4 in the manuscript.

Regarding the question in how far lesions in specific areas may modulate the 'categorical analysis' during the presentation of distractor words (FIX). Figure 7A illustrates that lesions in a large cluster in the anterior temporal lobe correspond to a decrease in relative fixation of the related words. This cluster is closely collocated to a cluster in which lesions correspond to a decrease in the difference between relative fixation of related minus fixation of the unrelated distractor words $(\Delta$ FIX rel-unrel $\downarrow)$. A much smaller cluster in which lesions correspond to increasing relative fixation on unrelated words (FIXunrel $\uparrow$ ) largely overlaps with parts of the two above clusters. The fact that lesser fixation on related words (FIXrel $\downarrow$ ) is the more prominent cluster confirms the intuitive prediction that difficulties to recognize the categorical relationship should mostly decrease preferential fixation of related words. This finding is complemented by a correlation of a large cluster in the anterior temporal lobe (anterior part of the ITG) with a decrease in the performance in the NVST test, assessing non-verbal semantic abilities (Figure 7C).

Regarding the interference effect on naming, the results suggest that lesions in a cluster of the posterior MTG and another cluster in the STG
/ planum polare correlate to smaller interference $(\Delta$ VOTrel-unrel $\downarrow$, Figure $7 \mathrm{~B})$. While the posterior cluster (MTG) is close to a cluster in which VOT for unrelated pictures increases (VOTunrel $\uparrow$ ), the cluster in the mid STG partially overlaps with a decrease in VOT for related items (VOTrel $\downarrow$ ).

(A)

FIXATION
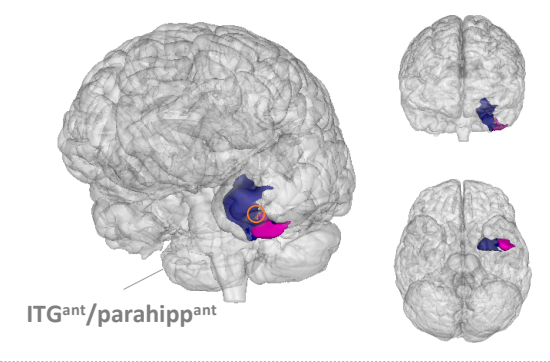

REL $\downarrow$

$\Delta$ (REL-UNR) $\downarrow$

UNR $\uparrow$

(B)

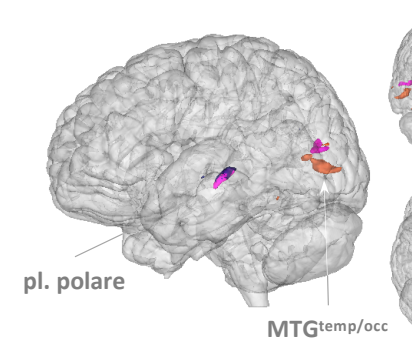

(C)

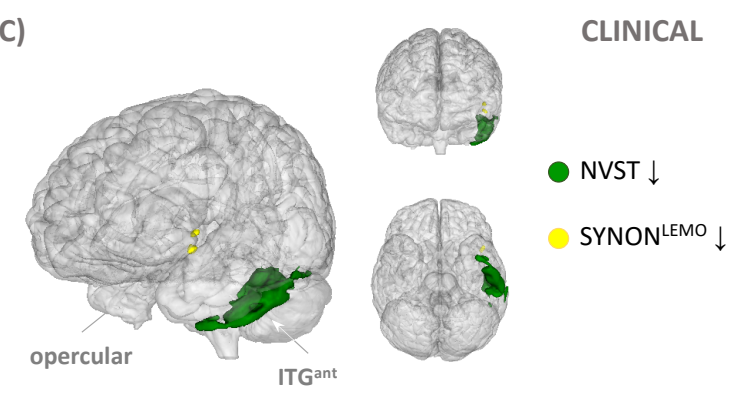

Voice Onset Time

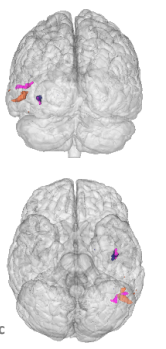

REL $\downarrow$

$\Delta($ REL-UNR) $\downarrow$

UNR $\uparrow$

Figure 7: Clusters (uncorrected) corresponding to the behavioral effects of VOT (VOTrel, VOTunr, $\Delta$ VOTrel-unrel) and FIX (FIXrel, FIXunr, $\triangle$ FIXrel-unr) as well as the clinical measures assessing semantic abilities (NVST and SYNONLEMO). Specifications of the clusters are provided in Table 4. 


\section{Discussion}

Lesions to the left hemispheric language network regularly interfere with the ability of prompt and correct retrieval of words. Clinically this results in the slowing of speech production and erroneous choice of lexical entries. One common type of such errors are semantic paraphasias leading to the substitution of a target word by a semantically related word (Schwartz, 2014). While it is the clinical goal of speech and language therapy (SLT) to restore fast and precise lexical retrieval in PWA, error patterns in these speakers may shed light on how our brain supports the remarkable ability of seemingly effortless language production. This in turn may allow for developing theoretically grounded therapy schemes also for confrontational naming training, a cornerstone of SLT in aphasia (Lorenz \& Ziegler, 2009; Off, Griffin, Spencer, \& Rogers, 2016).

In this vein we here investigate participants with a chronic lesion to the left hemispheric language network, all of whom showed overt aphasia in the acute stage of their disease and suffered from mild to moderate or residual aphasia at the time of testing. To enquire in how an acquired lesion impacts on lexico-semantic processing we used a novel semantic interference paradigm as previously investigated in neurotypical young participants (van Scherpenberg et al. 2020). Our results contribute to the question of how semantic context, generated by a set of 8 distractor words, affects language production after brain lesions causing aphasic deficits. We used a combined eye tracking and picture naming paradigm, to help provide evidence for two aspects of lexical retrieval: (i) the dynamics of analysis of a set of words regarding their semantic relation and (ii) efficiency and modulation of picture naming in a controlled semantic context. Besides implications for models of language production, the study adds to the growing body of work demonstrating feasibility and fruitfulness of complex language production paradigms in heterogenous cohorts of people with residual to moderate language impairment.

At the group level participants showed a strong semantic interference effect elicited by categorically related distractor words. The effect is significantly larger than that in neurotypical speakers. Notably, it is not correlated to clinically applied, linguistic tests including those targeting word-level deficits. In line with findings in the neurotypical cohort, the interference effect is independent of the number of distractor words (i.e., no difference between 3,4 , or 5 categorically related distractor words). Also in line with the findings in the neurotypical group, duration of fixation on the semantically related distractor words did not predict naming latency. Although overall naming latencies decreased significantly over the course of the experiment, the interference effect was stable across repetitions of the same picture across all trials. This contrasts findings in the neurotypical group, who showed interference only in the first cycle of naming. Interestingly, eye tracking additionally revealed that participants fixated equally long on all words. Thereby the preferential fixation of categorically related words in the neurotypical cohort is not preserved in the group of participants with a lesion to the language network. It is noteworthy, however, that participants who showed a preferential fixation on categorically related words performed better in the clinical test assessing overall semantic abilities (NVST).

The above behavioral results are complemented by findings of explorative lesion-behavior correlational analyses. Regarding overall semantic abilities lesions in the anterior temporal lobe (ATL) correlated with lesser performance in overall semantic (NVST $\downarrow$ ) and synonym judgment (SYNONLEMO $\downarrow$ ) abilities. The fact that lesions in similar clusters in the ATL correlate with smaller fixation preference for related compared to unrelated words ( $\Delta$ FIXrel-unrel $\downarrow$ ) suggests a common underlying neuronal network. With regard to the semantic interference effect on lexical retrieval, lesions in posterior MTG and in STG / planum polare, correlate with a smaller interference effects (as evidenced be voice onset times: $\Delta$ VOTrel-unrel $\downarrow$ ).

We will first briefly discuss two findings which are in line with the findings in the neurotypical population previously reported (van Scherpenberg et al., 2020). We then discuss the focus of the present paper, that is in how far a brain lesion elicits changes in semantic categorization and overt picture naming, while taking into account the results of our exploratory lesion behavior correlations.

\section{Naming latencies are independent of the number of categorically related distractors and the categorical fixation preference.}

Findings in the clinical and neurotypical group converge in that semantic interference is not 
affected by set size. For the clinical group, a significant effect is observed at 3,4 and 5 categorically related distractor words $(\sim 36 \mathrm{~ms}, \sim 38$ $\mathrm{ms}$ and $\sim 29 \mathrm{~ms}$, respectively), i.e., the effect did not increase with additional distractors. This supports the assumption that, reading of more categorically related distractor words does not induce more competition on target word retrieval. Besides number of overtly presented categorical distractors, results in both PWA and neurotypical groups do not speak for longer fixation on semantically related distractor words to increase interference. Neither number of overtly presented lexical competitors nor longer fixation seems to enhance lexical activation, which should have resulted in an increase in naming latency. Our null findings indicate that cohort activation does not critically depend on these two parameters, suggesting lexical competition is rather a function of semantic proximity and overall categorical cohort size (Aristei \& Abdel Rahman, 2013; Rabovsky, Schad, \& Abdel Rahman, 2016; Rose, Aristei, Melinger, \& Abdel Rahman, 2018; Vigliocco, Vinson, Damian, \& Levelt, 2002).

\section{PWA show large semantic interference, stable across naming repetitions}

The semantic interference effect observed in PWA is numerically much stronger than that of neurotypical participants ( $\sim 30 \mathrm{~ms}$ vs $\sim 10 \mathrm{~ms})$. Moreover, contrary to the neurotypical group, the effect in the clinical population is stable across naming repetitions of the same item. Despite an overall decrease in naming latency across repetitions ( $\sim 66 \mathrm{~ms}$ on average), the interference effect remained stable (no interaction of picture type and repetition). In the neurotypical group, both overall VOT and the interference effect $(\Delta$ VOTrel-unrel) decreased significantly. This suggests that the lesioned network shows larger 'vulnerability' of the correct lexical retrieval and cannot afford substantial 'learning' of the interference-suppression.

Previous evidence on semantic interference in PWA based on the picture-word-interference task is inconclusive. Some studies demonstrated a robust effect at the group level (Hashimoto \& Thompson, 2010; Piai \& Knight, 2017; Pino et al., n.d.), while others failed to report a significant effect in cohorts or in single case studies (Piai, Riès, \& Swick, 2016; Wilshire et al., 2007). Our study adds evidence of the effect in participants with aphasia over and above specific lexico-semantic abilities. The lack of significant correlations to clinical assessments on naming, word fluency or semantic abilities suggests that standard patholinguistic diagnostics are largely 'blind' to interference by categorical distractors. This may be of note for clinical perspectives because categorical distractor induced interference is qualitatively similar but substantially larger when compared to neurotypical participants.

The explorative VLSM analyses showed lesions in the left lateral-temporal cortex (STG and MTG) to decrease the interference effect. It highlights the region's key role in picture naming, more specifically the suggestion that temporal areas are essential for activation of the lexical target (Baldo, Arévalo, Patterson, \& Dronkers, 2013). A decrease in inference may seem counterintuitive at first, however co-activation of a lexical cohort can be assumed to be likewise affected by the lesion (Harvey \& Schnur, 2015; Henseler, Mädebach, Kotz, \& Jescheniak, 2014). Interestingly results of a brain stimulation study in neurotypical participants (Pisoni, Papagno, \& Cattaneo, 2012) have been interpreted in exactly this vein: less efficient activation of lexical entries reduces the number of co-activated lexical competitors, thereby reducing the inhibitory effect on target retrieval. Our exploratory lesion analysis supports this notion.

\section{Semantic interference does not depend on explicit acknowledgement of semantic category}

Without specific instruction neurotypical participants fixated longer on categorically related when compared to unrelated words in the distractor word set. This indicates an acknowledgement or explicit processing of the semantic category present in the word set. Interestingly, this "categorization effect" was not found in the current study. Participants with aphasia showed no fixation preference for categorically related words. The finding is particularly relevant in that is speaks for a nonstraightforward relationship between categorical semantic processing and lexical retrieval. This requires discussion.

Apart from the picture-word-interference task, blocked-cyclic-naming and continuous-naming paradigms elicit semantic interference and may shed light on the relationship between semantic and lexical processing. In the blocked cyclic naming paradigm, pictures are named consecutively either within homogenous blocks of 
one semantic category, or heterogeneous blocks of several semantic categories (Belke, Meyer, \& Damian, 2005; Belke \& Stielow, 2013; Crowther \& Martin, 2014; Schnur, Schwartz, Brecher, \& Hodgson, 2006). Here, semantic interference reliably appears in the comparison of naming latencies between the two naming settings: pictures are named more slowly in homogenous than in heterogenous blocks. By repeated retrieval of members of the same semantic category, their lexical representations form a strong cohort of mutual competitors, constantly inhibiting retrieval of the respective target lexical representation. Recently, it has been debated that the blocked cyclic naming paradigm leaves room for additional, task related strategies potentially influencing the effect: Because of the repetitively presented semantic categories, neurotypical participants become familiar with the items in the set which may allow them to bias the lexicalsemantic representations of the set members, increasing efficiency of lexical retrieval (Belke, 2017b, 2017a). This suggests that, in the blocked cyclic paradigm, participants are aware of the semantic relationship of the pictures they are naming. While this effect does not override the inhibition of naming latencies within blocks, it counteracts accumulation of interference across blocks. The assumption is supported by the fact that participants with lesions in the LPFC do exhibit stronger cumulative effects, i.e., stronger semantic interference also across blocks (Belke \& Stielow, 2013; Riès et al., 2015; Schnur et al., 2009). Lesions in this area, known to be involved in executive control functions and retrieval of semantic knowledge (Thompson-Schill, Bedny, \& Goldberg, 2005; Thompson-Schill, D'Esposito, Aguirre, \& Farah, 1997) may compromise the topdown bias of the lexical-semantic representations of the set members in the respective cycle.

In contrary, in the continuous naming paradigm (Howard et al., 2006; Oppenheim, Dell, \& Schwartz, 2010; Schnur, 2014), pictures are also named consecutively, but in seemingly random order with category members separated by pictures from other categories as well as fillers. Nevertheless, with each new member of a category that has to be named, latencies have been shown to cumulatively increase (see also Kuhlen \& Abdel Rahman, 2017; Rose \& Abdel Rahman, 2016). Even though participants are likely not aware of the categorical relationship of the pictures due to their seemingly random distribution, their relatedness nevertheless exerts an inhibitory function on naming latencies. The continuous naming paradigm therefore supports the notion that explicit processing of a semantic relationship is not necessary to induce interference.

The current study adds to this debate. On the one hand, our study demonstrates robust and large semantic picture-word interference induced by multiple semantic distractors, in a group of participants with chronic lesions in the left hemispheric language network including the temporal lobe and IFG. However, this effect is not dependent on explicit processing of the semantic category, which participants viewed as part of the circular word set beforehand. Participants fixated equally long on semantically related compared to unrelated words. This pattern is clearly different from our findings in neurotypical participants (van Scherpenberg et al., 2020) resembles findings in participants with semantic memory impairment. As reported by Seckin et al. (2016) and Faria et al. (2018) individuals with Semantic Dementia spent more time fixating on unrelated picture foils in a visual world paradigm, compared to neurotypical participants. Participants in the present study did not exhibit strong difficulties in semantic processing, as indicated by the clinical tests (LEMO Synonyms; NVST non-verbal semantics tasks; see Table 1). However, correlations between the experimental measures and the test scores revealed that the better participants performed in the NVST, the bigger the difference in fixation durations between category members and non-members, indicating a higher ability for semantic differentiation. Additionally, the explorative lesion-symptom correlations showed that both lower performance in the NVST and LEMO Synonyms task as well as a decreased difference in fixation durations correlated with lesions in the ATL. The ATL has been described to have the function of a semantic hub integrating multi-modal semantic information (e.g., Mesulam et al., 2009, 2013; Pobric et al., 2007). While interpretation of our VLSM analyses is tentative, results point to an involvement of the ATL in our modified pictureword interference paradigm. We suggest that lexical information from the written words is mapped on their (amodal) semantic correspondence, which would be the prerequisite to explicitly acknowledge their semantic categorical relationship. Lesions in the ATL may compromise this ability, while lexical co-activation, 
as a prerequisite for the interference effect is largely preserved.

Despite the absence of explicit semantic categorization, naming latencies were significantly delayed when the target picture was categorically related to the distractor word set. Moreover, the effect was even stronger than that in the neurotypical population, who showed a clear semantic categorization effect as evidenced by fixation durations. These findings underline the assumption that semantic information from lexically activated distractors (be it written distractor words or previously named pictures) is implicitly processed to activate further category members. Although somewhat speculative, the absence of a relationship between fontal (IFG) lesions and either aspect of the experimental task performance may point in the same direction. The implicitly activated category members in turn form a cohort of lexical competitors which inhibit target selection when the target is part of the same semantic category (lexical competition hypothesis, e.g., Abdel Rahman \& Melinger, 2009, 2019; La Heij et al., 2006; Melinger \& Abdel Rahman, 2013; Wheeldon \& Monsell, 1994).

It should be noted that in our paradigm the SOA, i.e. the time between first presentation of a distractor word and display of the target picture $(8$ s), was substantially longer than that in typical picture-word-interference tasks. We used the long SOA to ensure that each word was processed. On average participants fixated $\sim 720 \mathrm{~ms}$ on each word in the word set. Therefore, even if lexico-semantic processing was slowed in our clinical population, the exploration time of $8 \mathrm{~s}$ can be assumed sufficient to retrieve the semantic content of the words and activation of a cohort of competitors. In fact, the single case study by Wilshire et al. (2007) revealed (a trend towards) semantic interference only after enough time had passed for successful semantic activation of the distractor word (at SOA of +200 or $+400 \mathrm{~ms}$ ). We therefore suggest that the paradigm described here adds further evidence that semantic interference through lexical competition is independent of explicit processing or acknowledgement of semantic information of previously activated distractors of pictures. However, the fact that we do find interference for related compared to unrelated distractor words implies that implicit, automated semantic processing must have taken place for the distractor words to function as competitors inhibiting naming.

\section{Conclusion}

Taken together, the results of the current study add to the knowledge on semantic context effects during word retrieval in aphasia. We applied a complex novel multi-word picture-wordinterference paradigm combining both naming latency as well as eye movement measures, to examine the relationship between explicit processing of the semantic content of the distractor words and their inhibitory effect on the following naming task. We replicated a robust semantic interference effect from multiple simultaneously presented distractor words, which was numerically stronger than that of a neurotypical population in the same paradigm. Comparable to the results from the neurotypical population, the effect was similarly strong for the varying number of distractors in the word set and was not modulated by longer fixation durations to the semantically related distractor words. This leads us to conclude that beyond implicit semantic processing of the category, overtly adding members to the lexical cohort or longer processing of the lexical entries does not further modulate lexical competition during the word retrieval process. Moreover, the interference effect arose despite no explicit processing of the categorical relationship between the distractor words, i.e., despite the fact that participants fixated equally long on members and non-members of the semantic category present in the word set. This supports the assumption that implicit, automatic semantic activation upon reading the distractor words is sufficient to cause interference. Implicit semantic processing can be assumed to underly the interference effect in the continuous naming paradigm. In the blocked-cyclic naming paradigm, however, it has recently been debated that participants are likely aware of the semantic relationship of items in the homogenous blocks, which may in fact attenuate the effect across cycles. In our paradigm, a smaller difference in fixations on category members vs. non-members correlated with lower non-verbal semantic abilities (in the NVST task) and lesions in the ATL, implying an involvement of this amodal semantic hub in explicit semantic processing in this task. Future research could corroborate this implication by assessing the relationship of semantic processing and naming abilities in participants with Semantic Dementia, whose semantic competence is known to be strongly impaired, due to atrophy in the ATL. 


\section{References}

Abdel Rahman, R., \& Melinger, A. (2008). Enhanced phonological facilitation and traces of concurrent word form activation in speech production: An object-naming study with multiple distractors. Quarterly Journal of Experimental Psychology, 61(9), 1410-1440.

https://doi.org/10.1080/17470210701560724

Abdel Rahman, R., \& Melinger, A. (2009). Semantic context effects in language production: A swinging lexical network proposal and a review. Language and Cognitive Processes, 24(5), 713-734. https://doi.org/10.1080/01690960802597250

Abdel Rahman, R., \& Melinger, A. (2019). Semantic processing during language production: an update of the swinging lexical network. Language, Cognition and Neuroscience, 34(9), 1176-1192. https://doi.org/10.1080/23273798.2019.1599970

Aristei, S., \& Abdel Rahman, R. (2013). Semantic interference in language production is due to graded similarity, not response relevance. Acta Psychologica, 144, 571-582.

Aschenbrenner, S., Tucha, O., \& Lange, K. W. (2001). Regensburger Wortflüssigkeits-Test. (1, Ed.). Göttingen: Hogrefe.

Baldo, J. V, Arévalo, A., Patterson, J. P., \& Dronkers, N. F. (2013). Grey and white matter correlates of picture naming: Evidence from a voxel-based lesion analysis of the Boston Naming Test. Cortex, 49(3), 658-667.

https://doi.org/https://doi.org/10.1016/j.cortex .2012.03.001

Bates, D., Maechler, M., Bolker, B., \& Walker, S. (2015). Fitting Linear Mixed-Effects Models Using lme4. Journal of Statistical Software, 67(1), 1-48. https://doi.org/10.18637/jss.v067.i01

Belke, E. (2017a). Effects of lesions to the left lateral prefrontal cortex on task-specific top-down biases and response strategies in blocked-cyclic naming. Cognitive Neuropsychology, 34(1-2), 26-32. https://doi.org/10.1080/02643294.2017.1329200

Belke, E. (2017b). The role of task-specific response strategies in blocked-cyclic naming. Frontiers in Psychology, 7(JAN), 1-6. https://doi.org/10.3389/fpsyg.2016.01955

Belke, E., Meyer, A. S., \& Damian, M. F. (2005).

Refractory effects in picture naming as assessed in a semantic blocking paradigm. The Quarterly Journal of Experimental Psychology Section A, 58(4), 667-692. https:// doi.org/10.1080/02724980443000142

Belke, E., \& Stielow, A. (2013). Cumulative and noncumulative semantic interference in object naming: Evidence from blocked and continuous manipulations of semantic context. Quarterly Journal of Experimental Psychology, 66(11), 21352160.

https://doi.org/10.1080/17470218.2013.775318
Bloem, I., \& La Heij, W. (2003). Semantic facilitation and semantic interference in word translation: Implications for models of lexical access in language production. Journal of Memory and Language, 48(3), 468-488. https://doi.org/10.1016/S0749-596X(02)00503-X

Bloem, I., van den Boogaard, S., \& La Heij, W. (2004). Semantic facilitation and semantic interference in language production: Further evidence for the conceptual selection model of lexical access. Journal of Memory and Language, 51(2), 307-323. https://doi.org/10.1016/j.jml.2004.05.001

Boersma, P., \& Weenink, D. (2018). Praat: doing phonetics by computer. Retrieved from http://www.praat.org

Brainard, D. H. (1997). The Psychophysics Toolbox. Spatial Vision, 10(4), 433-436.

https://doi.org/doi:https://doi.org/10.1163/156 856897 X00357

Bürki, A., Elbuy, S., Madec, S., \& Vasishth, S. (2020). What did we learn from forty years of research on semantic interference? A Bayesian meta-analysis. Journal of Memory and Language, 114, 104125. https:/ / doi.org/10.1016/J.JML.2020.104125

Crowther, J. E., \& Martin, R. C. (2014). Lexical selection in the semantically blocked cyclic naming task: the role of cognitive control and learning. Frontiers in Human Neuroscience, 8(January), 1-20. https:// doi.org/10.3389/fnhum.2014.00009

Damian, M. F., \& Bowers, J. S. (2003). Locus of semantic interference in picture-word interference tasks. Psychonomic Bulletin and Review, 10(1), 111-117. https://doi.org/10.3758/BF03196474

de Zubicaray, G. I., \& Piai, V. (2019). Investigating the spatial and temporal components of speech production. In G. I. de Zubicaray \& N. O. Schiller (Eds.), The Oxford Handbook of Neurolinguistics (pp. 1-28). Oxford University Press.

Dell, G. S., Schwartz, M. F., Martin, N., Saffran, E. M., \& Gagnon, D. A. (1997). Lexical access in aphasics and nonaphasic speakers. Psychological Review, 104(4), 801-838.

DeMarco, A. T., \& Turkeltaub, P. E. (2018). A multivariate lesion symptom mapping toolbox and examination of lesion-volume biases and correction methods in lesion-symptom mapping. Human Brain Mapping, 39(11), 4169-4182. https:// doi.org/10.1002/hbm.24289

Faria, A. V., Race, D., Kim, K., \& Hillis, A. E. (2018). The eyes reveal uncertainty about object distinctions in semantic variant primary progressive aphasia. Cortex, 103, 372-381. https://doi.org/10.1016/j.cortex.2018.03.023

Glaser, W. R., \& Düngelhoff, F. (1984). The Time Course of Picture-Word Interference. Journal of Experimental Psychology: Human Perception and Performance, 10(5), 640-654.

Goodglass, H., \& Wingfield, A. (1997). Word-finding deficits in aphasia: Clinical symptomatology and 
brain-behavior relationships. In Anomia (pp. 325). San Diego, CA: Academic Press.

Gorno-Tempini, M. L., Hillis, A. E., Weintraub, S., Kertesz, A., Mendez, M., Cappa, S. F., ... Grossman, M. (2011). Classification of primary progressive aphasia and its variants. Neurology, 76(11), 1006-1014.

https://doi.org/10.1212/WNL.0b013e31821103e6

Harvey, D. Y., \& Schnur, T. T. (2015). Distinct loci of lexical and semantic access deficits in aphasia: Evidence from voxel-based lesion-symptom mapping and diffusion tensor imaging. Cortex, 67, 37-58.

https://doi.org/10.1016/j.cortex.2015.03.004

Hashimoto, N., \& Thompson, C. K. (2010). The use of the picture-word interference paradigm to examine naming abilities in aphasic individuals. Aphasiology, 24(5), 580-611. Retrieved from http:/ / 10.0.4.56/02687030902777567

Henseler, I., Mädebach, A., Kotz, S. A., \& Jescheniak, J. D. (2014). Modulating Brain Mechanisms Resolving Lexico-semantic Interference during Word Production: A Transcranial Direct Current Stimulation Study. Journal of Cognitive

Neuroscience, 26(7), 1403-1417. https://doi.org/10.1162/jocn

Hogrefe, K., Glindemann, R., Ziegler, W., \& Goldenberg, G. (n.d.). Nonverbaler Semantiktest (NVST). Göttingen: Hogrefe.

Howard, D., Nickels, L., Coltheart, M., \& Cole-Virtue, J. (2006). Cumulative semantic inhibition in picture naming: experimental and computational studies. Cognition, 100(3), 464-482. https://doi.org/10.1016/j.cognition.2005.02.006

Huber, W., Poeck, K., Weniger, D., \& Willmes, K. (1983). Aachener Aphasie Test (1st ed.). Göttingen: Hogrefe.

Huettig, F., Rommers, J., \& Meyer, A. S. (2011). Using the visual world paradigm to study language processing: A review and critical evaluation. Acta Psychologica, 137(2), 151-171.

https://doi.org/10.1016/j.actpsy.2010.11.003

Kohn, S. E., \& Goodglass, H. (1985). Picture-Naming in Aphasia. Brain and Language, 24, 266-283.

Kuhlen, A. K., \& Abdel Rahman, R. (2017). Having a task partner affects lexical retrieval: Spoken word production in shared task settings. Cognition, 166, 94-106. https://doi.org/10.1016/j.cognition.2017.05.024

Kuznetsova, A., Brockhoff, P. B., \& Haubo, R. (2016). lmerTest: Tests in Linear Mixed Effect Models. Retrieved from https://cran.rproject.org/package $=$ lmerTest

La Heij, W., Kuipers, J.-R., \& Starreveld, P. A. (2006). In Defense of the Lexical-Competition Account of Picture-Word Interference: A Comment on Finkbeiner and Caramazza (2006). Cortex, 42(7), 1028-1031. https://doi.org/10.1016/S00109452(08)70209-0
Levelt, W. J. M., Roelofs, A., \& Meyer, A. S. (1999). A Theory of Lexical Access in Speech Production. Behavioural and Brain Sciences, 22, 1-75. https:// doi.org/10.3115/992628.992631

Lorenz, A., \& Ziegler, W. (2009). Semantic vs. wordform specific techniques in anomia treatment: A multiple single-case study. Journal of Neurolinguistics, 22(6), 515-537. https://doi.org/10.1016/j.jneuroling.2009.05.003

Melinger, A., \& Abdel Rahman, R. (2013). Lexical selection is competitive: Evidence from indirectly activated semantic associates during picture naming. Journal of Experimental Psychology: Learning Memory and Cognition, 39(2), 348-364. https:// doi.org/10.1037/a0028941

Mesulam, M. M., Rogalski, E. J., Wieneke, C., Cobia, D., Rademaker, A., Thompson, C. K., \& Weintraub, S. (2009). Neurology of anomia in the semantic variant of primary progressive aphasia. Brain, 132(9), 2553-2565. https://doi.org/10.1093/brain/awp138

Mesulam, M. M., Wieneke, C., Hurley, R. S., Rademaker, A., Thompson, C. K., Weintraub, S., \& Rogalski, E. J. (2013). Words and objects at the tip of the left temporal lobe in primary progressive aphasia. Brain, 136(2), 601-618. https://doi.org/10.1093/brain/aws336

Meyer, A. S., \& Schriefers, H. (1991). Phonological facilitation in picture-word interference experiments: Effects of stimulus onset asynchrony and types of interfering stimuli. Journal of Experimental Psychology: Learning, Memory, and Cognition, 17(6), 1146-1160. https:// doi.org/10.1037/0278-7393.17.6.1146

Mirman, D., Chen, Q., Zhang, Y., Wang, Z., Faseyitan, O. K., Coslett, H. B., \& Schwartz, M. F. (2015). Neural organization of spoken language revealed by lesion-symptom mapping. Nature Communications, 6, 1-9. https://doi.org/10.1038/ncomms7762

Morey, R. D. (2008). Confidence Intervals from Normalized Data: A correction to Cousineau (2005). Tutorials in Quantitative Methods for Psychology, 4(2), 61-64. https://doi.org/10.20982/tqmp.04.2.p061

Nozari, N. (2020). Neural Basis of Word Production. In The Oxford Handbook of The Mental Lexicon.

Off, C. A., Griffin, J. R., Spencer, K. A., \& Rogers, M. A. (2016). The impact of dose on naming accuracy with persons with aphasia. Aphasiology, 30(9), 983-1011. https:// doi.org/10.1080/02687038.2015.1100705

Oppenheim, G. M., Dell, G. S., \& Schwartz, M. F. (2010). The dark side of incremental learning: A model of cumulative semantic interference during lexical access in speech production. Cognition, 114(2), 227-252.

https://doi.org/10.1016/j.cognition.2009.09.007

Piai, V., \& Knight, R. T. (2017). Lexical selection with 
competing distractors: Evidence from left temporal lobe lesions. Psychonomic Bulletin and Review, 1-8. https://doi.org/10.3758/s13423-0171301-0

Piai, V., Riès, S. K., \& Swick, D. (2016). Lesions to Lateral Prefrontal Cortex Impair Lexical Interference Control in Word Production. Frontiers in Human Neuroscience, 9(January), 1-13. https://doi.org/10.3389/fnhum.2015.00721

Pino, D., Mädebach, A., Jescheniak, J. D., Regenbrecht, F., \& Obrig, H. (n.d.). BONEs not CATs attract DOGs: Semantic context effects on picture naming in lesioned language network.

Pisoni, A., Papagno, C., \& Cattaneo, Z. (2012). Neural correlates of the semantic interference effect: New evidence from transcranial direct current stimulation. Neuroscience, 223, 56-67. https://doi.org/https://doi.org/10.1016/j.neuro science.2012.07.046

Pobric, G., Jefferies, E., \& Lambon Ralph, M. A. (2007). Anterior temporal lobes mediate semantic representation: Mimicking semantic dementia by using rTMS in normal participants. Proceedings of the National Academy of Sciences, 104(50), 2013720141. https://doi.org/10.1073/pnas.0707383104

$R$ Core Team. (2016). R: A language and environment for statistical computing. Vienna, Austria: R Foundation for Statistical Computing. Retrieved from https://www.r-project.org/

Rabovsky, M., Schad, D. J., \& Abdel Rahman, R. (2016). Language production is facilitated by semantic richness but inhibited by semantic density: Evidence from picture naming. Cognition, 146, 240-244. https://doi.org/10.1016/j.cognition.2015.09.016

Riès, S. K., Karzmark, C. R., Navarrete, E., Knight, R. T., \& Dronkers, N. F. (2015). Specifying the role of the left prefrontal cortex in word selection. Brain and Language, 149, 135-147. https:/ / doi.org/10.1016/J.BANDL.2015.07.007

Roelofs, A. (1992). A spreading-activation theory of lemma retrieval in speaking. Cognition, 42, 107142.

Rose, S. B., \& Abdel Rahman, R. (2016). Semantic similarity promotes interference in the continuous naming paradigm: behavioural and electrophysiological evidence. Language, Cognition and NeuroscienceCognitive Processes, 32(1), 55-68.

Rose, S. B., Aristei, S., Melinger, A., \& Abdel Rahman, R. (2018). The Closer They Are, the More They Interfere: Semantic Similarity of Word Distractors Increases Competition in Language Production. Journal of Experimental Psychology: Learning Memory and Cognition. https://doi.org/10.1037/xlm0000592

Roux, F., Armstrong, B. C., \& Carreiras, M. (2017). Chronset: An automated tool for detecting speech onset. Behavior Research Methods, 49(5), 1864-1881. https://doi.org/10.3758/s13428-016-0830-1
Schnur, T. T. (2014). The persistence of cumulative semantic interference during naming. Journal of Memory and Language, 75, 27-44. https://doi.org/10.1016/j.jml.2014.04.006

Schnur, T. T., Schwartz, M. F., Brecher, A., \& Hodgson, C. (2006). Semantic interference during blockedcyclic naming: Evidence from aphasia. Journal of Memory and Language, 54(2), 199-227. https://doi.org/10.1016/j.jml.2005.10.002

Schnur, T. T., Schwartz, M. F., Kimberg, D. Y., Hirshorn, E., Coslett, H. B., \& Thompson-Schill, S. L. (2009). Localizing interference during naming: Convergent neuroimaging and neuropsychological evidence for the function of Broca's area. Proceedings of the National Academy of Sciences, 106(1), 322 LP - 327. https://doi.org/10.1073/pnas.0805874106

Schriefers, H., Meyer, A. S., \& Levelt, W. J. M. (1990). Exploring the time-course of lexical access in production: Picture-word interference studies. Journal of Memory and Language, 29(1), 86-102.

Schwartz, M. F. (2014). Theoretical analysis of word production deficits in adult aphasia. Philosophical Transactions of the Royal Society B: Biological Sciences, 369(1634), 20120390. https://doi.org/10.1098/rstb.2012.0390

Seckin, M., Mesulam, M. M., Voss, J. L., Huang, W., Rogalski, E. J., \& Hurley, R. S. (2016). Am I looking at a cat or a dog? Gaze in the semantic variant of primary progressive aphasia is subject to excessive taxonomic capture. Journal of Neurolinguistics, 37, 68-81. https://doi.org/10.1016/j.jneuroling.2015.09.003

Stadie, N., Cholewa, J., \& De Bleser, R. (2013). LEMO 2.0 - Lexikon modellorientiert. Hofheim: NATVerlag.

Starreveld, P. A., \& La Heij, W. (1996). Time-course analysis of semantic and orthographic context effects in picture naming. Journal of Experimental Psychology: Learning, Memory, and Cognition, 22(4), 896-918. https://doi.org/10.1037/02787393.22.4.896

Starreveld, P. A., \& La Heij, W. (2017). Picture-word interference is a Stroop effect: A theoretical analysis and new empirical findings. Psychonomic Bulletin and Review, 24, 721-733. https:// doi.org/10.3758/s13423-016-1167-6

Thompson-Schill, S. L., Bedny, M., \& Goldberg, R. F. (2005). The frontal lobes and the regulation of mental activity. Current Opinion in Neurobiology, 15(2), 219-224.

https://doi.org/https://doi.org/10.1016/j.conb. 2005.03.006

Thompson-Schill, S. L., D'Esposito, M., Aguirre, G. K., \& Farah, M. J. (1997). Role of left inferior prefrontal cortex in retrieval of semantic knowledge: a reevaluation. Proceedings of the National Academy of Sciences of the United States of America, 94(26), 14792-14797. 
https://doi.org/10.1073/pnas.94.26.14792

van Renswoude, D. R., Raijmakers, M. E. J., Koornneef, A., Johnson, S. P., Hunnius, S., \& Visser, I. (2018). Gazepath: An eye-tracking analysis tool that accounts for individual differences and data quality. Behavior Research Methods, 50(2), 834-852. https://doi.org/10.3758/s13428-017-0909-3

van Scherpenberg, C., Abdel Rahman, R., \& Obrig, H. (2020). A novel multi-word paradigm for investigating semantic context effects in language production. PLoS ONE, 15(4), 1-20. https://doi.org/10.1371/journal.pone.0230439

Vigliocco, G., Vinson, D. P., Damian, M. F., \& Levelt, W. J. M. (2002). Semantic distance effects on object and action naming. Cognition, 85(3), 61-69. https://doi.org/10.1016/S0010-0277(02)00107-5

Vigliocco, G., Vinson, D. P., Lewis, W., \& Garrett, M. F. (2004). Representing the meanings of object and action words: The featural and unitary semantic space hypothesis. Cognitive Psychology, 48(4), 422488.

https://doi.org/10.1016/j.cogpsych.2003.09.001
Wheeldon, L. R., \& Monsell, S. (1994). Inhibition of Spoken Word Production by Priming a Semantic Competitor. Journal of Memory and Language, 33(3), 332-356. https://doi.org/10.1006/JMLA.1994.1016

Wilshire, C. E., Keall, L. M., Stuart, E. J., \& O'Donnell, D. J. (2007). Exploring the dynamics of aphasic word production using the picture-word interference task: A case study. Neuropsychologia, 45, 939-953. https://doi.org/10.1016/J.NEUROPSYCHOLOG IA.2006.08.026

Yee, E., Blumstein, S. E., \& Sedivy, J. C. (2008). LexicalSemantic Activation in Broca's and Wernicke's Aphasia: Evidence from Eye Movements. Journal of Cognitive Neuroscience, 20(4), 592-612. https://doi.org/10.1162/jocn.2008.20056

Zhang, Y., Kimberg, D. Y., Coslett, H. B., Schwartz, M. F., \& Wang, Z. (2014). Multivariate lesionsymptom mapping using support vector regression. Hum Brain Mapp, 35(12), 5861-5876. https://doi.org/10.1002/hbm.22590 


\section{Appendix A. Stimuli}

Table A1: List of stimuli

\begin{tabular}{|c|c|c|c|c|c|c|}
\hline Category & Items & & & & & \\
\hline hoofed animals & $\begin{array}{l}\text { Reh } \\
\text { (deer) }\end{array}$ & $\begin{array}{l}\text { Pferd } \\
\text { (horse) }\end{array}$ & $\begin{array}{l}\text { Esel } \\
\text { (donkey) }\end{array}$ & $\begin{array}{l}\text { Schaf } \\
\text { (sheep) }\end{array}$ & $\begin{array}{l}\text { Kamel } \\
\text { (camel) }\end{array}$ & $\begin{array}{l}\text { Ziege } \\
\text { (goat) }\end{array}$ \\
\hline fruits & $\begin{array}{l}\text { Apfel } \\
\text { (apple) }\end{array}$ & $\begin{array}{l}\text { Birne } \\
\text { (pear) }\end{array}$ & $\begin{array}{l}\text { Traube } \\
\text { (grape) }\end{array}$ & $\begin{array}{l}\text { Erdbeere } \\
\text { (strawberry) }\end{array}$ & $\begin{array}{l}\text { Kirsche } \\
\text { (cherry) }\end{array}$ & $\begin{array}{l}\text { Orange } \\
\text { (orange) }\end{array}$ \\
\hline seating furniture & $\begin{array}{l}\text { Sofa } \\
\text { (couch) }\end{array}$ & $\begin{array}{l}\text { Stuhl } \\
\text { (chair) }\end{array}$ & $\begin{array}{l}\text { Hocker } \\
\text { (stool) }\end{array}$ & $\begin{array}{l}\text { Sessel } \\
\text { (armchair) }\end{array}$ & $\begin{array}{l}\text { Bank } \\
\text { (bench) }\end{array}$ & $\begin{array}{l}\text { Thron } \\
\text { (throne) }\end{array}$ \\
\hline carpenter's tools & $\begin{array}{l}\text { Hammer } \\
\text { (hammer) }\end{array}$ & $\begin{array}{l}\text { Säge } \\
\text { (saw) }\end{array}$ & $\begin{array}{l}\text { Schraube } \\
\text { (screw) }\end{array}$ & $\begin{array}{l}\text { Axt } \\
\text { (axe) }\end{array}$ & $\begin{array}{l}\text { Zange } \\
\text { (pliers) }\end{array}$ & $\begin{array}{l}\text { Bohrer } \\
\text { (drill) }\end{array}$ \\
\hline face parts & $\begin{array}{l}\text { Auge } \\
\text { (eye) }\end{array}$ & $\begin{array}{l}\text { Nase } \\
\text { (nose) }\end{array}$ & $\begin{array}{l}\text { Mund } \\
\text { (mouth) }\end{array}$ & $\begin{array}{l}\text { Ohr } \\
\text { (ear) }\end{array}$ & $\begin{array}{l}\text { Kinn } \\
\text { (chin) }\end{array}$ & $\begin{array}{l}\text { Haare } \\
\text { (hair) }\end{array}$ \\
\hline street vehicles & $\begin{array}{l}\text { Auto } \\
\text { (car) }\end{array}$ & $\begin{array}{l}\text { Lastwagen } \\
\text { (truck) }\end{array}$ & $\begin{array}{l}\text { Motorrad } \\
\text { (motorcycle) }\end{array}$ & $\begin{array}{l}\text { Kutsche } \\
\text { (carriage) }\end{array}$ & $\begin{array}{l}\text { Bus } \\
\text { (bus) }\end{array}$ & $\begin{array}{l}\text { Traktor } \\
\text { (tractor) }\end{array}$ \\
\hline $\begin{array}{l}\text { upper boddy } \\
\text { clothing }\end{array}$ & $\begin{array}{l}\text { Mantel } \\
\text { (coat) }\end{array}$ & $\begin{array}{l}\text { Jacke } \\
\text { (jacket) }\end{array}$ & $\begin{array}{l}\text { Pullover } \\
\text { (sweater) }\end{array}$ & $\begin{array}{l}\text { Hemd } \\
\text { (shirt) }\end{array}$ & $\begin{array}{l}\text { T-Shirt } \\
\text { (t-shirt) }\end{array}$ & $\begin{array}{l}\text { Bluse } \\
\text { (blouse) }\end{array}$ \\
\hline
\end{tabular}

\section{Appendix B. Fixation durations}

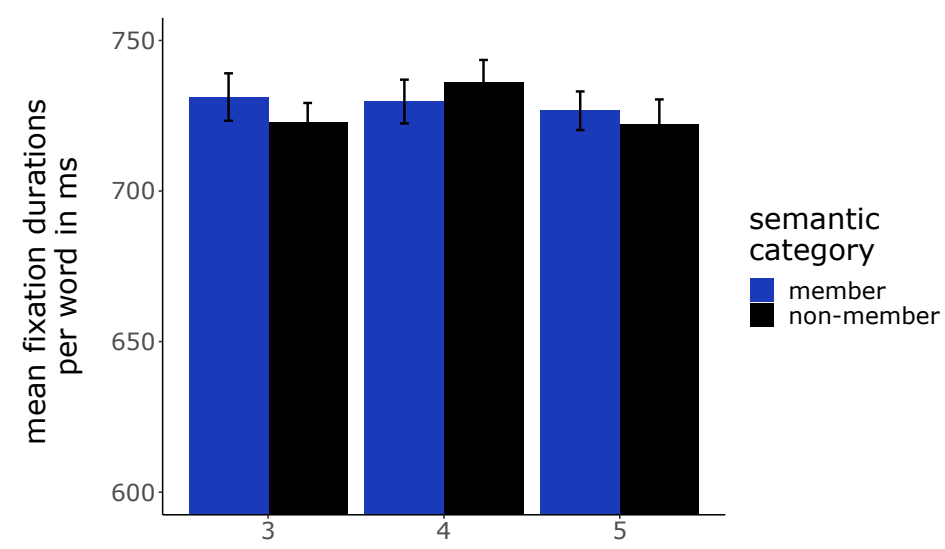

number of category members

Figure B1: Mean fixation durations per word for category members vs. non-members. 


\section{Appendix C. Correlations with clinical linguistic measures}

Table C1: Correlations between experimental variables and linguistic test scores.

\begin{tabular}{llll} 
linguistic test score & experimental effect & Spearman's rho & p-value \\
\hline \multirow{2}{*}{ LEMO: Reading } & $\Delta$ fixation durations & 0.235 & 0.229 \\
& semantic interference & -0.093 & 0.637 \\
\hline \multirow{2}{*}{ LEMO: Synonyms } & $\Delta$ fixation durations & 0.244 & 0.21 \\
& semantic interference & -0.207 & 0.29 \\
\hline \multirow{2}{*}{ AAT: Naming } & $\Delta$ fixation durations & -0.218 & 0.318 \\
& semantic interference & -0.26 & 0.231 \\
\multirow{2}{*}{ fluency: animals } & $\Delta$ fixation durations & -0.12 & 0.586 \\
& semantic interference & -0.235 & 0.281 \\
\hline \multirow{2}{*}{ NVST } & $\Delta$ fixation durations & 0.463 & $\mathbf{0 . 0 1 3}$ \\
& semantic interference & 0.099 & 0.617
\end{tabular}

